\title{
Constructing the AdS dual of a Fermi liquid: AdS black holes with Dirac hair
}

\author{
Mihailo Čubrović, Jan Zaanen and Koenraad Schalm \\ Institute Lorentz for Theoretical Physics, Leiden University, \\ P.O. Box 9506, Leiden 2300RA, The Netherlands \\ E-mail: cubrovic@lorentz.leidenuniv.nl, jan@lorentz.leidenuniv.nl, \\ kschalm@lorentz.leidenuniv.nl
}

ABSTRACT: We provide evidence that the holographic dual to a strongly coupled charged Fermi liquid has a non-zero fermion density in the bulk. We show that the pole-strength of the stable quasiparticle characterizing the Fermi surface is encoded in the AdS probability density of a single normalizable fermion wavefunction in AdS. Recalling Migdal's theorem which relates the pole strength to the Fermi-Dirac characteristic discontinuity in the number density at $\omega_{F}$, we conclude that the AdS dual of a Fermi liquid is described by occupied on-shell fermionic modes in AdS. Encoding the occupied levels in the total spatially averaged probability density of the fermion field directly, we show that an AdS ReissnerNordström black hole in a theory with charged fermions has a critical temperature, at which the system undergoes a first-order transition to a black hole with a non-vanishing profile for the bulk fermion field. Thermodynamics and spectral analysis support that the solution with non-zero AdS fermion-profile is the preferred ground state at low temperatures.

Keywords: Black Holes in String Theory, AdS-CFT Correspondence, Holography and condensed matter physics (AdS/CMT) 


\section{Contents}

1 Introduction $\quad 1$

2 From Green's function to AdS/CFT rules for a Fermi liquid 4

2.1 The AdS dual of a stable Fermi liquid: applying Migdal's relation holographically

$\begin{array}{lll}2.1 .1 & \text { Boundary conditions and normalizability } & 11\end{array}$

3 An AdS black hole with Dirac hair 12

$\begin{array}{lll}3.1 \text { Thermodynamics } & 16\end{array}$

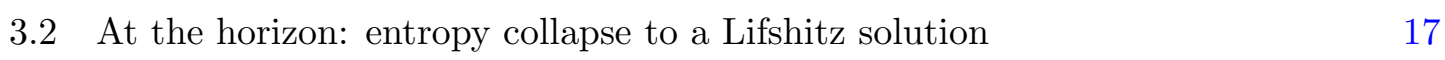

$\begin{array}{lll}3.3 & \text { A BH with Dirac hair } & 18\end{array}$

$\begin{array}{lll}\text { 3.3.1 Finite fermion density solutions in AdS-RN } & 19\end{array}$

3.3.2 Finite fermion density in AdSS 22

3.4 Confirmation from fermion spectral functions 23

4 Discussion and conclusion $\quad 24$

\section{Introduction}

Fermionic quantum criticality is thought to be an essential ingredient in the full theory of high $T_{c}$ superconductivity $[1,2]$. The cleanest experimental examples of quantum criticality occur in heavy-fermion systems rather than high $T_{c}$ cuprates, but the experimental measurements in heavy fermions raise equally confounding theoretical puzzles [3]. Most tellingly, the resistivity scales linearly with the temperature from the onset of superconductivity up to the crystal melting temperature [4] and this linear scaling is in conflict with single correlation length scaling at criticality [5]. The failure of standard perturbative theoretical methods to describe such behavior is thought to indicate that the underlying quantum critical system is strongly coupled [6, 7].

The combination of strong coupling and scale-invariant critical dynamics makes these systems an ideal arena for the application of the AdS/CFT correspondence: the wellestablished relation between strongly coupled conformal field theories (CFT) and gravitational theories in anti-de Sitter (AdS) spacetimes. An AdS/CFT computation of singlefermion spectral functions - which are directly experimentally accessible via AngleResolved Photoemission Spectroscopy [8-10] — bears out this promise of addressing fermionic quantum criticality [11-15] (see also [16, 17]). The AdS/CFT single fermion spectral function exhibits distinct sharp quasiparticle peaks, associated with the formation of a Fermi surface, emerging from a scale-free state. The fermion liquid which this 
Fermi surface captures is generically singular: it has either a non-linear dispersion or nonquadratic pole strength $[11,13]$. The precise details depend on the parameters of the AdS model.

From the AdS gravity perspective, peaks with linear dispersion correspond to the existence of a stable charged fermionic quasinormal mode in the spectrum of a charged AdS black hole. The existence of a stable charged bosonic quasinormal mode is known to signal the onset of an instability towards a new ground state with a pervading Bose condensate extending from the charged black hole horizon to the boundary of AdS. The dual CFT description of this charged condensate is spontaneous symmetry breaking as in a superfluid and a conventional superconductor [18-21]. For fermionic systems empirically the equivalent robust $T=0$ ground state is the Landau Fermi Liquid - the quantum ground state of a system with a finite number of fermions. The existence of a stable fermionic quasinormal mode suggests that an AdS dual of a finite fermion density state exists.

Here we shall make a step towards the set of AdS/CFT rules for CFTs with a finite fermion density. The essential ingredient will be Migdal's theorem, which relates the characteristic jump in fermion occupation number at the energy $\omega_{F}$ of the highest occupied state to the pole strength of the quasiparticle. The latter we know from the spectral function analysis and its AdS formulation is therefore known. Using this, we can show that the fermion number discontinuity is encoded in the probability density of the normalizable wavefunction of the dual AdS fermion field.

This shows that the AdS dual of a Fermi liquid is given by a system with occupied fermionic states in the bulk. The Fermi liquid is clearly not a scale invariant state, but any such states will have energy, momentum/pressure and charge and will change the interior geometry from AdS to something else. Which particular (set of) state(s) is the right one, it does not yet tell us, as this conclusion relies only on the asymptotic behavior of fermion fields near the AdS boundary. Here we shall take the simplest such state: a single fermion. ${ }^{1}$ Constructing first a set of equations in terms of the spatially averaged density, we find the associated backreacted asymptotically AdS solution. This approximate solution is already good enough to solve several problems of principle:

- A charged AdS black hole in the presence of charged fermionic modes has a critical temperature below which fermionic Dirac "hair" forms. For our effective single fermion solution, the derivative of the free energy has the characteristic discontinuity of a first order transition. In AdS/CFT this has to be the case: In contrast to bosonic quasinormal modes, a fermionic quasinormal mode can never cause a linear instability indicative of a continuous phase transition. In the language of spectral functions, the pole of the retarded Green's function can never cross to the upper-half plane [13]. ${ }^{2}$ The absence of a perturbative instability between this conjectured Dirac "black hole hair" solution and the "bald" charged AdS black hole can be explained if the transition is a first order gas-liquid transition. The existence of first order transition follows from a thermodynamic analysis of the free energy rather than a spectral analysis of small fluctuations.

\footnotetext{
${ }^{1}$ These solutions are therefore the AdS extensions of [22-25].

${ }^{2}$ Ref. [41] argues that the instability can be second order.
} 
- This solution with finite fermion profile is the preferred ground state at low temperatures compared to the bare charged AdS black hole. The latter is therefore a false vacuum in a theory with charged fermions. Confusing a false vacuum with the true ground state can lead to anomalous results. Indeed the finite temperature behavior of fermion spectral functions in AdS Reissner-Nordström, exhibited in the combination of the results of $[11,13]$ and [12], shows strange behavior. The former $[11,13]$ found sharp quasiparticle peaks at a frequency $\omega_{F}=0$ in natural AdS units, whereas the latter [12] found sharp quasiparticle peaks at finite Fermi energy $\omega_{F} \neq 0$. As we will show, both peaks in fact describe the same physics: the $\omega_{F} \neq 0$ peak is a finite temperature manifestation of (one of the) $\omega=0$ peaks in [13]. Its shift in location at finite temperature is explained by the existence of the nearby true finite fermion density ground state, separated by a potential barrier from the AdS Reissner-Nordström solution.

- The solution we construct here only considers the backreaction on the electrostatic potential. We show, however, that the gravitational energy density diverges at the horizon. This ought to be, as one expects the infrared geometry to change due to fermion profile. The charged AdS-black hole solution corresponds to a CFT system in a state with large ground state entropy. This is the area of the extremal black-hole horizon at $T=0$. Systems with large ground-state entropy are notoriously unstable to collapse to a low-entropy state, usually by spontaneous symmetry breaking. In a fermionic system it should be the collapse to the Fermi liquid. The final state will generically be a geometry that asymptotes to Lifschitz type, i.e. the background breaks Lorentz-invariance and has a double-pole horizon with vanishing area, as expounded in [26]. Indeed the gravitational energy density diverges at the horizon in a similar way as other systems that are known to gravitationally backreact to a Lifshitz solution. The fully backreacted geometry includes important separate physical aspects - it is relevant to the stability and scaling properties of the Fermi liquid and will be considered in a companion article.

The Dirac hair solution thus captures the physics one expects of the dual of a Fermi liquid. We have based its construction on a derived set of AdS/CFT rules to describe systems at finite fermion density. Qualitatively the result is as expected: one also needs occupied fermionic states in the bulk. Next to our effective single fermion approximation, another simple candidate is the backreacted AdS-Fermi-gas [26]/electron star [27] which appeared during the course of this work. ${ }^{3}$ The difference between the two approaches are the assumptions used to reduce the interacting Fermi system to a tractable solution. Ideally, one should carefully track all the fermion wavefunctions as in the recent article [38]. As explained in [31] the Fermi-gas and the single Dirac field are the two "local" approximations to the generic non-local multiple fermion system in the bulk, in very different regimes of applicability. The electron-star/Fermi-gas is considered in the Thomas-Fermi limit where the microscopic charge of the constituent fermions is sent to zero keeping the overall charge

\footnotetext{
${ }^{3}$ See also [28, 29]. An alternative approach to back-reacting fermions is [30].
} 
fixed, whereas the single Dirac field clearly is the 'limit' where the microscopic charge equals the total charge in the system. This is directly evident in the spectral functions of both systems. The results presented here show that each pole in the CFT spectral function corresponds to a unique occupied Fermi state in the bulk; the electron star spectra show a parametrically large number of poles [31-33], whereas the Dirac hair state has a single quasiparticle pole by construction. The AdS-Dirac-hair black hole derived here therefore has the benefit of a direct connection with a unique Fermi liquid state in the CFT. This is in fact the starting point of our derivation.

In the broader context, the existence of both the Dirac hair and backreacted Fermi gas solution is not a surprise. It is a manifestation of universal physics in the presence of charged AdS black holes. The results here, and those of [11, 13, 26, 27], together with the by now extensive literature on holographic superconductors, i.e. Bose condensates, show that at sufficiently low temperature in units of the black-hole charge, the electric field stretching to AdS-infinity causes a spontaneous discharge of the bulk vacuum outside of the horizon into the charged fields of the theory - whatever their nature. The positively charged excitations are repelled by the black hole, but cannot escape to infinity in AdS and they form a charge cloud hovering over the horizon. The negatively charged excitations fall into the black-hole and neutralize the charge, until one is left with an uncharged black hole with a condensate at finite $T$ or a pure asymptotically AdS-condensate solution at $T=0$. As $[26,27]$ and we show, the statistics of the charged particle do not matter for this condensate formation, except in the way it forms: bosons superradiate and fermions nucleate. The dual CFT perspective of this process is "entropy collapse". The final state therefore has negligible ground state entropy and is stable. The study of charged black holes in AdS/CFT is therefore a novel way to understand the stability of charged interacting matter which holds much promise.

\section{From Green's function to AdS/CFT rules for a Fermi liquid}

We wish to show how a solution with finite fermion number - a Fermi liquid - is encoded in AdS. The exact connection and derivation will require a review of what we have learned of Dirac field dynamics in AdS/CFT through Green's functions analysis. The defining signature of a Fermi liquid is a quasi-particle pole in the (retarded) fermion propagator,

$$
G_{R}=\frac{Z}{\omega-\mu_{R}-v_{F}\left(k-k_{F}\right)}+\text { regular }
$$

Phenomenologically a non-zero residue at the pole, $Z$, also known as the pole strength, is the indicator of a Fermi liquid state. Migdal famously related the pole strength to the occupation number discontinuity at the pole $\omega=0$.

$$
Z=\lim _{\epsilon \rightarrow 0}\left[n_{F}(\omega-\epsilon)-n_{F}(\omega+\epsilon)\right]
$$

where

$$
n_{F}(\omega)=\int d^{2} k f_{F D}\left(\frac{\omega}{T}\right) \operatorname{Im} G_{R}(\omega, k) .
$$


with $f_{F D}$ the Fermi-Dirac distribution function. Vice versa, a Fermi liquid with a FermiDirac jump in occupation number at the Fermi energy $\omega_{F}=0$ has a low-lying quasiparticle excitation. Using our knowledge of fermionic spectral functions in AdS/CFT we shall first relate the pole-strength $Z$ to known AdS quantities. Then using Migdal's relation, the dual of a Fermi liquid is characterized by an asymptotically AdS solution with non-zero value for these very objects.

The Green's functions derived in AdS/CFT are those of charged fermionic operators with scaling dimension $\Delta$, dual to an AdS Dirac field with mass $m=\Delta-\frac{d}{2}$. We shall focus on $d=2+1$ dimensional CFTs. In its gravitational description this Dirac field is minimally coupled to $3+1$ dimensional gravity and electromagnetism with action

$$
S=\int d^{4} x \sqrt{-g}\left[\frac{1}{2 \kappa^{2}}\left(R+\frac{6}{L^{2}}\right)-\frac{1}{4} F_{M N}^{2}-\bar{\Psi}(\not D+m) \Psi\right] .
$$

For zero background fermions, $\Psi=0$, a spherically symmetric solution is a charged $\mathrm{AdS}_{4}$ black-hole background

$$
\begin{aligned}
d s^{2} & =\frac{L^{2} \alpha^{2}}{z^{2}}\left(-f(z) d t^{2}+d x^{2}+d y^{2}\right)+\frac{L^{2}}{z^{2}} \frac{d z^{2}}{f(z)}, \\
f(z) & =(1-z)\left(1+z+z^{2}-q^{2} z^{3}\right), \\
A_{0}^{(b g)} & =2 q \alpha(z-1) .
\end{aligned}
$$

Here $A_{0}^{(b g)}$ is the time-component of the $\mathrm{U}(1)$-vector-potential, $L$ is the AdS radius and the temperature and chemical potential of the black hole equal

$$
T=\frac{\alpha}{4 \pi}\left(3-q^{2}\right), \quad \mu_{0}=-2 q \alpha,
$$

where $q$ is the black hole charge.

To compute the Green's functions we need to solve the Dirac equation in the background of this charged black hole:

$$
e_{A}^{M} \Gamma^{A}\left(D_{M}+i \mathrm{e} g A_{M}\right) \Psi+m \Psi=0
$$

where the vielbein $e_{A}^{M}$, covariant derivative $D_{M}$ and connection $A_{M}$ correspond to the fixed charged AdS black-hole metric and electrostatic potential (2.4) Denoting $A_{0}=\Phi$ and taking the standard AdS-fermion projection onto $\Psi_{ \pm}=\frac{1}{2}\left(1 \pm \Gamma^{Z}\right) \Psi$, the Dirac equation reduces to

$$
\left(\partial_{z}+\mathcal{A}_{ \pm}\right) \Psi_{ \pm}=\mp \mathcal{T} \Psi_{\mp}
$$

with

$$
\begin{aligned}
\mathcal{A}_{ \pm} & =-\frac{1}{2 z}\left(3-\frac{z f^{\prime}}{2 f}\right) \pm \frac{m L}{z \sqrt{f}} \\
\mathcal{F} & =\frac{i(-\omega+g \Phi)}{\alpha f} \gamma^{0}+\frac{i}{\alpha \sqrt{f}} k_{i} \gamma^{i} .
\end{aligned}
$$


Here $\gamma^{\mu}$ are the 2+1-dimensional Dirac matrices, obtained after decomposing the $3+1$ dimensional $\Gamma^{\mu}$-matrices.

Explicitly the Green's function is extracted from the behavior of the solution to the Dirac equation at the AdS-boundary. The boundary behavior of the bulk fermions is

$$
\begin{aligned}
& \Psi_{+}(\omega, k ; z)=A_{+} z^{\frac{3}{2}-m}+B_{+} z^{\frac{5}{2}+m}+\ldots, \\
& \Psi_{-}(\omega, k ; z)=A_{-} z^{\frac{5}{2}-m}+B_{-} z^{\frac{3}{2}+m}+\ldots,
\end{aligned}
$$

where $A_{ \pm}(\omega, k), B_{ \pm}(\omega, k)$ are not all independent but related by the Dirac equation at the boundary

$$
A_{-}=-\frac{i \mu}{(2 m-1)} \gamma^{0} A_{+}, \quad B_{+}=-\frac{i \mu}{(2 m+1)} \gamma^{0} B_{-} .
$$

The CFT Green's function then equals [11, 12, 34]

$$
G_{R}=\lim _{z \rightarrow 0} z^{-2 m} \frac{\Psi_{-}(z)}{\Psi_{+}(z)}-\text { singular }=\frac{B_{-}}{A_{+}} .
$$

In other words $B_{-}$is the CFT response to the (infinitesimal) source $A_{+}$. Since in the Green's function the fermion is a fluctuation, the functions $\Psi_{ \pm}(z)$ are now probe solutions to the Dirac equation in a fixed gravitational and electrostatic background (for ease of presentation we are considering $\Psi_{ \pm}(z)$ as numbers instead of two-component vectors). The boundary conditions at the horizon/AdS interior determine which Green's function one considers, e.g. infalling horizon boundary conditions yield the retarded Green's function. For non-zero chemical potential this fermionic Green's function can have a pole signalling the presence of a Fermi surface. This pole occurs precisely for a (quasi-)normalizable mode, i.e. a specific energy $\omega_{F}$ and momentum $k_{F}$ where the external source $A_{+}(\omega, k)$ vanishes (for infalling boundary conditions at the horizon).

Knowing that the energy of the quasinormal mode is always $\omega_{F}=0$ [11] and following [13], we expand $G_{R}$ around $\omega=0$ as:

$$
G_{R}(\omega)=\frac{B_{+}^{(0)}+\omega B^{(1)_{+}}+\ldots}{A_{+}^{(0)}+\omega A_{+}^{(1)}+\ldots} .
$$

A crucial point is that in this expansion we are assuming that the pole will correspond to a stable quasiparticle, i.e. there are no fractional powers of $\omega$ less than unity in the expansion around $\omega_{F}=0$ [13]. Fermions in AdS/CFT are of course famous for allowing more general pole-structures corresponding to Fermi-surfaces without stable quasiparticles [13], but those Green's functions are not of the type (2.1) and we shall therefore not consider them here. The specific Fermi momentum $k_{F}$ associated with the Fermi surface is the momentum value for which the first $\omega$-independent term in the denominator vanishes $A_{+}^{(0)}\left(k_{F}\right)=0$ - for this value of $k=k_{F}$ the presence of a pole in the Green's functions at $\omega=0$ is manifest. Writing $A_{+}^{(0)}=a_{+}\left(k-k_{F}\right)+\ldots$ and comparing with the standard quasi-particle propagator,

$$
G_{R}=\frac{Z}{\omega-\mu_{R}-v_{F}\left(k-k_{F}\right)}+\text { regular }
$$


we read off that the pole-strength equals

$$
Z=B_{-}^{(0)}\left(k_{F}\right) / A_{+}^{(1)}\left(k_{F}\right) .
$$

We thus see that a non-zero pole-strength is ensured by a non-zero value of $B_{-}\left(\omega=0, k=k_{F}\right)$ - the "response" without corresponding source as $A^{(0)}\left(k_{F}\right) \equiv 0$. Quantitatively the pole-strength also depends on the value of $\left.A_{+}^{(1)}\left(k_{F}\right) \equiv \partial_{\omega} A_{+}\left(k_{F}\right)\right|_{\omega=0}$, which is always finite. This is not a truly independent parameter, however. The size of the pole-strength has only a relative meaning w.r.t. to the integrated spectral density. This normalization of the pole strength is a global parameter rather than an AdS boundary issue. We now show this by proving that $A_{+}^{(1)}\left(k_{F}\right)$ is inversely proportional to $B_{-}^{(0)}\left(k_{F}\right)$ and hence $Z$ is completely set by $B_{-}^{(0)}\left(k_{F}\right)$, i.e. $Z \sim\left|B_{-}^{(0)}\left(k_{F}\right)\right|^{2}$. Consider a transform $\widetilde{W}\left(\Psi_{+, A}, \Psi_{+, B}\right)$ of the Wronskian $W\left(\Psi_{+, A}, \Psi_{+, B}\right)=\Psi_{+, A} \partial_{z} \Psi_{+, B}-\left(\partial_{z} \Psi_{+, A}\right) \Psi_{+, B}$ for two solutions to the second order equivalent of the Dirac equation for the field $\Psi_{+}$

$$
\left(\partial_{z}^{2}+P(z) \partial_{z}+Q_{+}(z)\right) \Psi_{+}=0
$$

that is conserved (detailed expressions for $P(z)$ and $Q_{+}(z)$ are given in eq. (2.21)):

$$
\widetilde{W}\left(\Psi_{+, A}(z), \Psi_{+, B}(z), z ; z_{0}\right)=\exp \left(\int_{z_{0}}^{z} P(z)\right) W\left(\Psi_{+, A}(z), \Psi_{+, B}(z)\right), \quad \partial_{z} \widetilde{W}=0 .
$$

Here $z_{0}$ is the infinitesimal distance away from the boundary at $z=0$ which is equivalent to the $U V$-cutoff in the CFT. Setting $k=k_{F}$ and choosing for $\Psi_{+, A}=A_{+} z^{3 / 2-m} \sum_{n=0}^{\infty} a_{n} z^{n}$ and $\Psi_{+, B}=B_{+} z^{5 / 2+m} \sum_{n=0}^{\infty} b_{n} z^{n} r$ the real solutions which asymptote to solutions with $B_{+}\left(\omega, k_{F}\right)=0$ and $A_{+}\left(\omega, k_{F}\right)=0$ respectively, but for a value of $\omega$ infinitesimally away from $\omega_{F}=0$, we can evaluate $\widetilde{W}$ at the boundary to find, ${ }^{4}$

$$
\widetilde{W}=z_{0}^{3}(1+2 m) A_{+} B_{+}=\mu z_{0}^{3} A_{+} B_{-}
$$

The last step follows from the constraint (2.10) where the reduction from two-component spinors to functions means that $\gamma^{0}$ is replaced by one of its eigenvalues $\pm i$. Taking the derivative of $\widetilde{W}$ at $\omega=0$ for $k=k_{F}$ and expanding $A_{+}\left(\omega, k_{F}\right)$ and $B_{-}\left(\omega, k_{F}\right)$ as in (2.12), we can solve for $A_{+}^{(1)}\left(k_{F}\right)$ in terms of $B_{-}^{(0)}\left(k_{F}\right)$ and arrive at the expression for the pole strength $Z$ in terms of $\left|B_{-}^{(0)}\left(k_{F}\right)\right|^{2}$ :

$$
Z=\frac{\mu z_{0}^{3}}{\left.\partial_{\omega} \widetilde{W}\right|_{\omega=0, k=k_{F}}}\left|B_{-}^{(0)}\left(k_{F}\right)\right|^{2}
$$

Because $\partial_{\omega} \widetilde{W}$, as $\widetilde{W}$, is a number that is independent of $z$, this expression emphasizes that it is truly the nonvanishing subleading term $B_{-}^{(0)}\left(\omega_{F}, k_{F}\right)$ which sets the pole strength, up to a normalization $\partial_{\omega} \widetilde{W}$ which is set by the fully integrated spectral density. This integration is always UV-cut-off dependent and the explicit $z_{0}$ dependence should therefore

\footnotetext{
${ }^{4} P(z)=-3 / z+\ldots$ near $z=0$
} 
not surprise us. ${ }^{5}$ We should note that, unlike perturbative Fermi liquid theory, $Z$ is a dimensionful quantity of mass dimension $2 m+1=2 \Delta-2$, which illustrates more directly its scaling dependence on the UV-energy scale $z_{0}$. At the same time $Z$ is real, as it can be shown that both $\left.\partial_{\omega} \widetilde{W}\right|_{\omega=0, k=k_{F}}=\mu z_{0}^{3} A_{+}^{(1)} B_{-}^{(0)}$ and $B_{-}^{(0)}$ are real [13].

\subsection{The AdS dual of a stable Fermi liquid: applying Migdal's relation holographically}

We have thus seen that a solution with nonzero $B_{-}\left(\omega_{F}, k_{F}\right)$ whose corresponding external source vanishes (by definition of $\omega_{F}, k_{F}$ ), is related to the presence of a quasiparticle pole in the CFT. Through Migdal's theorem its pole strength is related to the presence of a discontinuity of the occupation number, and this discontinuity is normally taken as the characteristic signature of the presence of a Fermi Liquid. Qualitatively we can already infer that an AdS gravity solution with non-vanishing $B_{-}\left(\omega_{F}, k_{F}\right)$ corresponds to a Fermi Liquid in the CFT. We thus seek solutions to the Dirac equation with vanishing external source $A_{+}$but non-vanishing response $B_{-}$coupled to electromagnetism (and gravity). The construction of the AdS black hole solution with a finite single fermion wavefunction is thus analogous to the construction of a holographic superconductor [19] with the role of the scalar field now taken by a Dirac field of mass $m$.

This route is complicated, however, by the spinor representation of the Dirac fields, and the related fermion doubling in AdS. Moreover, relativistically the fermion Green's function is a matrix and the pole strength $Z$ appears in the time-component of the vector projection $\operatorname{Tr} i \gamma^{i} G$. As we take this and the equivalent jump in occupation number to be the signifying characteristic of a Fermi liquid state in the CFT, it would be much more direct if we can derive an AdS radial evolution equation for the vector-projected Green's function and hence the occupation number discontinuity directly. From the AdS perspective is also more convenient to work with bilinears such as Green's functions, since the Dirac fields always couple pairwise to bosonic fields.

To do so, we start again with the two decoupled second order equations equivalent to the Dirac equation (2.7)

$$
\left(\partial_{z}^{2}+P(z) \partial_{z}+Q_{ \pm}(z)\right) \Psi_{ \pm}=0
$$

\footnotetext{
${ }^{5}$ Using that $\widetilde{W}$ is conserved, one can e.g. compute it at the horizon. There each solution $\Psi_{+, A}\left(\omega, k_{F} ; z\right)$, $\Psi_{+, B}\left(\omega, k_{F} ; z\right)$ is a linear combination of the infalling and outgoing solution

$$
\begin{aligned}
& \Psi_{+, A}(z)=\bar{\alpha}(1-z)^{-1 / 4+\imath \omega / 4 \pi T}+\alpha(1-z)^{-1 / 4-\imath \omega / 4 \pi T}+\ldots \\
& \Psi_{+, B}(z)=\bar{\beta}(1-z)^{-1 / 4+\imath \omega / 4 \pi T}+\beta(1-z)^{-1 / 4-\imath \omega / 4 \pi T}+\ldots
\end{aligned}
$$

yielding a value of $\partial_{\omega} \widetilde{W}$ equal to $(P(z)=1 / 2(1-z)+\ldots$ near $z=1)$

$$
\partial_{\omega} \widetilde{W}=\frac{i}{2 \pi T} \mathcal{N}\left(z_{0}\right)(\bar{\alpha} \beta-\bar{\beta} \alpha)
$$

with $\mathcal{N}\left(z_{0}\right)=\exp \int_{z_{0}}^{z} d z\left[P(z)-\frac{1}{2(1-z)}\right]$
} 
with

$$
\begin{aligned}
P(z) & =\left(\mathcal{A}_{-}+\mathcal{A}_{+}\right)-\left[\partial_{z}, \mathcal{F}\right] \frac{\mathcal{F}}{T^{2}} \\
Q_{ \pm}(z) & =\mathcal{A}_{-} \mathcal{A}_{+}+\left(\partial_{z} \mathcal{A}_{ \pm}\right)-\left[\partial_{z}, \mathcal{H}\right] \frac{\mathcal{F}}{T^{2}} \mathcal{A}_{ \pm}+T^{2}
\end{aligned}
$$

Note that both $P(z)$ and $Q_{ \pm}(z)$ are matrices in spinor space. The general solution to this second order equation - with the behavior at the horizon/interior appropriate for the Green's function one desires - is a matrix valued function $\left(M_{ \pm}(z)\right)_{\beta}^{\alpha}$ and the field $\Psi_{ \pm}(z)$ equals $\Psi_{ \pm}(z)=M_{ \pm}(z) \Psi_{ \pm}^{(\text {hor })}$. Due to the first order nature of the Dirac equation the horizon values $\Psi_{ \pm}^{\text {(hor) }}$ are not independent but related by a $z$-independent matrix $S \Psi_{+}^{\text {(hor) }}=$ $\Psi_{-}^{(\text {hor })}$, which can be deduced from the near-horizon behavior of $(2.10)$; specifically $S=\gamma^{0}$. One then obtains the Green's function from the on-shell boundary action (see e.g. [12, 35])

$$
S_{\mathrm{bnd}}=\oint_{z=z_{0}} d^{d} x \bar{\Psi}_{+} \Psi_{-}
$$

as follows: Given a boundary source $\zeta_{+}$for $\Psi_{+}(z)$, i.e. $\Psi_{+}\left(z_{0}\right) \equiv \zeta_{+}$, one concludes that $\Psi_{+}^{(\text {hor })}=M_{+}^{-1}\left(z_{0}\right) \zeta_{+}$and thus $\Psi_{+}(z)=M_{+}(z) M_{+}^{-1}\left(z_{0}\right) \zeta_{+}, \Psi_{-}(z)=M_{-}(z) S M_{+}^{-1}\left(z_{0}\right) \zeta_{+}$. Substituting these solutions into the action gives

$$
S_{\mathrm{bnd}}=\oint_{z=z_{0}} d^{d} x \bar{\zeta}_{+} M_{-}\left(z_{0}\right) S M_{+}^{-1}\left(z_{0}\right) \zeta_{+}
$$

The Green's function is obtained by differentiating w.r.t. $\bar{\zeta}_{+}$and $\zeta_{+}$and discarding the conformal factor $z_{0}^{2 m}$ with $m$ the AdS mass of the Dirac field (one has to be careful for $m L>1 / 2$ with analytic terms [35])

$$
G=\lim _{z_{0} \rightarrow 0} z_{0}^{-2 m} M_{-}\left(z_{0}\right) S M_{+}^{-1}\left(z_{0}\right) .
$$

Since $M_{ \pm}(z)$ are determined by evolution equations in $z$, it is clear that the Green's function itself is also determined by an evolution equation in $z$, i.e. there is some function $G(z)$ which reduces in the limit $z \rightarrow 0$ to $z_{0}^{2 m} G$. One obvious candidate is the function

$$
G^{(\mathrm{obv})}(z)=M_{-}(z) S M_{+}^{-1}(z) .
$$

Using the original Dirac equations one can see that this function obeys the non-linear evolution equation

$$
\partial_{z} G^{(\mathrm{obv})}(z)=-\mathcal{A}_{-} G^{(\mathrm{obv})}(z)-\mathscr{T} M_{+} S M_{+}^{-1}+\mathcal{A}_{+} G^{(\mathrm{obv})}(z)+G^{(\mathrm{obv})}(z) \mathcal{T} G^{(\mathrm{obv})}(z) .
$$

This is the approach used in [11], where a specific choice of momenta is chosen such that $M_{+}$commutes with $S$. For a generic choice of momenta, consistency requires that one also considers the evolution equation for $M_{+}(z) S M_{+}^{-1}(z)$.

There is, however, another candidate for the extension $G(z)$ which is based on the underlying boundary action. Rather than extending the kernel $M_{-}\left(z_{0}\right) M_{+}^{-1}\left(z_{0}\right)$ of the boundary action we extend the constituents of the action itself, based on the individual 
fermion wavefunctions $\Psi_{ \pm}(z)=M_{ \pm}(z) S^{\frac{1}{2} \mp \frac{1}{2}} M_{+}^{-1}\left(z_{0}\right)$. We define an extension of the matrix $G(z)$ including an expansion in the complete set $\Gamma^{I}=\left\{\mathbb{1}, \gamma^{i}, \gamma^{i j}, \ldots, \gamma^{i_{1}, i_{d}}\right\}\left(\right.$ with $\left.\gamma^{4}=i \gamma^{0}\right)$

$$
G^{I}(z)=\bar{M}_{+}^{-1}\left(z_{0}\right) \bar{M}_{+}(z) \Gamma^{I} M_{-}(z) S M_{+}^{-1}\left(z_{0}\right), \quad G^{I}\left(z_{0}\right)=\Gamma^{I} G\left(z_{0}\right)
$$

where $\bar{M}=i \gamma^{0} M^{\dagger} i \gamma^{0}$. Using again the original Dirac equations, this function obeys the evolution equation

$\partial_{z} G^{I}(z)=-\left(\overline{\mathcal{A}}_{+}+\mathcal{A}_{-}\right) G^{I}(z)-\bar{M}_{+, 0}^{-1} \bar{M}_{-}(z) \overline{\mathcal{T}} \Gamma^{I} M_{-}(z) S M_{+, 0}^{-1}+\bar{M}_{+, 0}^{-1} \bar{M}_{+}(z) \Gamma^{I} \mathcal{T} M_{+}(z) S M_{+, 0}^{-1}$

Recall that $\mathcal{T} \gamma^{i_{1} \ldots i_{p}}=\mathcal{T}^{\left[i_{1}\right.} \gamma^{\left.\ldots i_{p}\right]}+\mathcal{T}_{j} \gamma^{j i_{1} \ldots i_{p}}$. It is then straightforward to see that for consistency, we also need to consider the evolution equations of

$$
\mathcal{J}_{+}^{I}=\bar{M}_{+, 0}^{-1} \bar{M}_{+}(z) \Gamma^{I} M_{+}(z) S M_{+, 0}^{-1}, \quad \mathcal{J}_{-}^{I}=\bar{M}_{+, 0}^{-1} \bar{M}_{-}(z) \Gamma^{I} M_{-}(z) S M_{+, 0}^{-1}
$$

and

$$
\bar{G}^{I}=\bar{M}_{+, 0}^{-1} \bar{M}_{-}(z) \Gamma^{I} M_{+}(z) S M_{+, 0}^{-1}
$$

They are

$$
\begin{aligned}
\partial_{z} \mathcal{J}_{+}^{i_{1} \ldots i_{p}}(z)= & -2 \operatorname{Re}\left(\mathcal{A}_{+}\right) \mathcal{J}_{+}^{i_{1} \ldots i_{p}}-\overline{\mathcal{T}}^{\left[i_{1}\right.} \bar{G}^{\left.i_{2} \ldots i_{p}\right]}(z) \\
& -\overline{\mathcal{T}}_{j} \bar{G}^{j i_{1} \ldots i_{p}}(z)-G^{\left[i_{1} \ldots i_{p-1}\right.}(z) \mathcal{T}^{\left.i_{p}\right]}-G^{i_{1} \ldots i_{p} j}(z) \mathcal{T}_{j} \\
\partial_{z} \mathcal{J}_{-}^{i_{1} \ldots i_{p}}(z)= & -2 \operatorname{Re}\left(\mathcal{A}_{-}\right) \mathcal{J}_{-}^{i_{1} \ldots i_{p}}+\overline{\mathcal{T}}^{\left[i_{1}\right.} G^{\left.i_{2} \ldots i_{p}\right]}(z) \\
& +\overline{\mathcal{T}}_{j} G^{j i_{1} \ldots i_{p}}(z)+\bar{G}^{\left[i_{1} \ldots i_{p-1}\right.}(z) \mathcal{T}^{\left.i_{p}\right]}+\bar{G}^{i_{1} \ldots i_{p} j}(z) \mathcal{T}_{j} \\
\partial_{z} \bar{G}^{i_{1} \ldots i_{p}}(z)= & -\left(\overline{\mathcal{A}}_{-}+\mathcal{A}_{+}\right) \bar{G}^{i_{1} \ldots i_{p}}-\overline{\mathcal{T}}^{\left[i_{1}\right.} \mathcal{J}_{+}^{\left.i_{2} \ldots i_{p}\right]}(z) \\
& -\overline{\mathcal{T}}_{j} \mathcal{J}_{+}^{j i_{1} \ldots i_{p}}(z)-\mathcal{J}_{-}^{\left[i_{1} \ldots i_{p-1}\right.}(z) \mathcal{T}^{\left.i_{p}\right]}+\mathcal{J}_{-}^{i_{1} \ldots i_{p} j}(z) \mathcal{T}_{j}
\end{aligned}
$$

The significant advantage of these functions $G^{I}, \bar{G}^{I}, \mathcal{J}_{ \pm}^{I}$ is that the evolution equations are now linear. This approach may seem overly complicated. However, if the vector $\mathcal{T}^{i}$ happens to only have a single component nonzero, then the system reduces drastically to the four fields $\mathcal{J}_{ \pm}^{i}, G^{\mathbb{1}}, \bar{G}^{\mathbb{1}}$. We shall see below that a similar drastic reduction occurs, when we consider only spatially and temporally averaged functions $J^{I}=\int d t d^{2} x \mathcal{J}_{ \pm}^{I}$.

Now the two extra currents $\mathcal{J}_{ \pm}^{I}$ have a clear meaning in the CFT. The current $G^{I}(z)$ reduces by construction to $\Gamma^{I}$ times the Green's function $G^{\mathbb{1}}\left(z_{0}\right)$ on the boundary, and clearly $\bar{G}^{I}(z)$ is its hermitian conjugate. The current $\mathcal{J}_{+}^{I}$ reduces at the boundary to $\mathcal{J}_{+}^{I}=\Gamma^{I} M_{+, 0} S M_{+, 0}^{-1}$. Thus $\mathcal{J}_{+}^{I}$ sets the normalization of the linear system (2.29). The interesting current is the current $\mathcal{J}_{-}^{I}$. Using that $\bar{S}=\bar{S}^{-1}$, it can be seen to reduce on the boundary to the combination $\overline{\mathcal{J}}_{+}^{\mathbb{G}} \bar{G}^{\mathbb{1}} \Gamma^{I} G^{\mathbb{1}}$. Thus, $\left(\overline{\mathcal{J}}_{+}^{\mathbb{1}}\right)^{-1} \mathcal{J}_{-}^{\mathbb{1}}$ is the norm squared of the Green's function, i.e. the probability density of the off-shell process.

For an off-shell process or a correlation function the norm-squared has no real functional meaning. However, we are specifically interested in solutions in the absence of an external source, i.e. the on-shell correlation functions. In that case the analysis is quite different. The on-shell condition is equivalent to choosing momenta to saturate the pole in the Green's function, i.e. it is precisely choosing dual AdS solutions whose leading external source $A_{ \pm}$ 
vanishes. Then $M_{+}$and $M_{-}$are no longer independent, but $M_{+, 0}=\delta B_{+} / \delta \Psi_{+}^{(\text {hor })}=$ $-\frac{i \mu \gamma^{0}}{2 m+1} M_{-, 0} S$. As a consequence all boundary values of $\mathcal{J}_{-}^{I}\left(z_{0}\right), G^{I}\left(z_{0}\right), \bar{G}^{I}\left(z_{0}\right)$ become proportional; specifically using $S=\gamma^{0}$ one has that

$$
\left.\mathcal{J}_{-}^{0}\left(z_{0}\right)\right|_{\text {on-shell }}=\left.\frac{(2 m+1)}{\mu} \gamma^{0} G^{\mathbb{1}}\left(z_{0}\right)\right|_{\text {on-shell }}
$$

is the "on-shell" Green's function. Now, the meaning of the on-shell correlation function is most evident in thermal backgrounds. It equals the density of states $\rho(\omega(k))=-\frac{1}{\pi} \operatorname{Im} G_{R}$ times the Fermi-Dirac distribution [36]

$$
\left.\operatorname{Tr} i \gamma^{0} G_{F}^{t}\left(\omega_{\text {bare }}(k), k\right)\right|_{\text {on-shell }}=2 \pi f_{F D}\left(\frac{\omega_{\text {bare }}(k)-\mu}{T}\right) \rho\left(\omega_{\text {bare }}(k)\right)
$$

For a Fermi liquid with the defining off-shell Green's function $(2.1) \omega_{\text {bare }}\left(k_{F}\right)-\mu \equiv \omega=0$ and $\rho\left(\omega_{\text {bare }}(k)\right)=Z_{z_{0}} \delta^{2}\left(k-k_{F}\right) \delta(\omega)+\ldots$. Thus we see that the boundary value of $\left.\mathcal{J}_{-}^{(0)}\left(z_{0}\right)\right|_{\text {on-shell }}=Z f_{F D}(0) \delta^{3}(0)$ indeed captures the pole strength directly times a product of distributions. This product of distributions can be absorbed in setting the normalization. An indication that this is correct is that the determining equations for $G^{I}, \bar{G}^{I}, \mathcal{J}_{ \pm}^{I}$ remain unchanged if we multiply $G^{I}, \bar{G}^{I}, \mathcal{J}_{ \pm}^{I}$ on both sides with $M_{+, 0}$. If $M_{+, 0}$ is unitary it is just a similarity transformation. However, from the definition of the Green's function, one can see that this transformation precisely removes the pole. This ensures that we obtain finite values for $G^{I}, \bar{G}^{I}, \mathcal{J}_{ \pm}^{I}$ at the specific pole-values $\omega_{F}, k_{F}$ where the distributions would naively blow up.

\subsubsection{Boundary conditions and normalizability}

We have shown that a normalizable solution to $\mathcal{J}_{-}^{0}$ from the equations (2.29) correctly captures the pole strength directly. However, 'normalizable' is still defined in terms of an absence of a source for the fundamental Dirac field $\Psi_{ \pm}$rather than the composite fields $\mathcal{J}_{ \pm}^{I}$ and $G^{I}$. One would prefer to determine normalizability directly from the boundary behavior of the composite fields. This can be done. Under the assumption that the electrostatic potential $\Phi$ is regular, i.e.

$$
\Phi=\mu-\rho z+\ldots
$$

the "connection" $\mathcal{T}^{I}$ is subleading to the connection $\mathcal{A}$ near $z=0$. Thus the equations of motion near $z=0$ do not mix the various $\mathcal{J}_{ \pm}^{I}, G^{I}$ and the composite fields behave as

$$
\begin{aligned}
\mathcal{J}_{+}^{I} & =j_{3-2 m}^{I} z^{3-2 m}+j_{4_{+}}^{I} z^{4}+j_{5+2 m}^{I} z^{5+2 m}+\ldots, \\
\mathcal{J}_{-}^{I} & =j_{5-2 m}^{I} z^{5-2 m}+j_{4-}^{I} z^{4}+j_{3+2 m}^{I} z^{3+2 m}+\ldots, \\
G^{I} & =I_{4-2 m}^{I} z^{4-2 m}+I_{3}^{I} z^{3}+I_{4+2 m}^{I} z^{4+2 m}+I_{5}^{I} z^{5}+\ldots,
\end{aligned}
$$

with the identification

$$
\begin{array}{lll}
j_{3-2 m}^{I}=\bar{A}_{+} \Gamma^{I} A_{+}, & j_{4_{+}}^{I}=\bar{A}_{+} \Gamma^{I} B_{+}+\bar{B}_{+} \Gamma^{I} A_{+}, & j_{5+2 m}^{I}=\bar{B}_{+} \Gamma^{I} B_{+}, \\
j_{3+2 m}^{I}=\bar{A}_{-} \Gamma^{I} A_{-}, & j_{4_{-}}^{I}=\bar{A}_{-} \Gamma^{I} B_{-}+\bar{B}_{-} \Gamma^{I} A_{-}, & j_{5-2 m}^{I}=\bar{B}_{-} \Gamma^{I} B_{-}, \\
I_{4-2 m}^{I}=\bar{A}_{+} \Gamma^{I} A_{-}, & I_{3}^{I}=\bar{A}_{+} \Gamma^{I} B_{-}, & I_{4+2 m}=\bar{B}_{+} \Gamma^{I} B_{-}, \quad I_{5}^{I}=\bar{B}_{+} \Gamma^{I} A_{-} .
\end{array}
$$


A 'normalizable' solution in $\mathcal{J}_{-}^{I}$ and thus $\mathcal{J}_{-}^{0}$ is therefore defined by the vanishing of both the leading and the subleading term.

\section{An AdS black hole with Dirac hair}

Having determined a set of AdS evolution equations and boundary conditions that compute the pole strength $Z$ directly through the currents $\mathcal{J}_{-}^{(0)}(z)$ and $G^{I}(z)$, we can now try to construct the AdS dual of a system with finite fermion density, including backreaction. As we remarked in the beginning of section 2.1, the demand that the solutions be normalizable means that the construction of the AdS black hole solution with a finite single fermion wavefunction is analogous to the construction of a holographic superconductor [19] with the role of the scalar field now taken by the Dirac field. The starting point therefore is the charged $\mathrm{AdS}_{4}$ black-hole background (2.4) and we should show that at low temperatures this AdS Reissner-Nordström black hole is unstable towards a solution with a finite Dirac profile. We shall do so in a simplified "large charge" limit where we ignore the gravitational dynamics, but as is well known from holographic superconductor studies (see e.g. [19-21]) this limit already captures much of the essential physics. In a companion article [37] we will construct the full backreacted ground state including the gravitational dynamics.

In this large charge non-gravitational limit the equations of motion for the action (2.3) reduce to those of $\mathrm{U}(1)$-electrodynamics coupled to a fermion with charge $g$ in the background of this black hole:

$$
\begin{aligned}
D_{M} F^{M N} & =i g e_{A}^{N} \bar{\Psi} \Gamma^{A} \Psi \\
0 & =e_{A}^{M} \Gamma^{A}\left(D_{M}+i \operatorname{eg} A_{M}\right) \Psi+m \Psi .
\end{aligned}
$$

Thus the vielbein $e_{A}^{M}$ and and covariant derivative $D_{M}$ remain those of the fixed charged AdS black hole metric (2.4), but the vector-potential now contains a background piece $A_{0}^{(b g)}$ plus a first-order piece $A_{M}=A_{M}^{(b g)}+A_{M}^{(1)}$, which captures the effect of the charge carried by the fermions.

Following our argument set out in previous section that it is more convenient to work with the currents $\mathcal{J}_{ \pm}^{I}(z), G^{I}(z)$ instead of trying to solve the Dirac equation directly, we shall first rewrite this coupled non-trivial set of equations of motion in terms of the currents while at the same time using symmetries to reduce the complexity. Although a system at finite fermion density need not be homogeneous, the Fermi liquid ground state is. It therefore natural to make the ansatz that the final AdS solution is static and preserves translation and rotation along the boundary. As the Dirac field transforms non-trivially under rotations and boosts, we cannot make this ansatz in the strictest sense. However, in some average sense which we will make precise, the solution should be static and translationally invariant. Then translational and rotational invariance allow us to set $A_{i}=0$, $A_{z}=0$, whose equations of motions will turn into contraints for the remaining degrees of freedom. Again denoting $A_{0}=\Phi$, the equations reduce to the following after the projection 
onto $\Psi_{ \pm}=\frac{1}{2}\left(1 \pm \Gamma^{Z}\right) \Psi$

$$
\begin{aligned}
\partial_{z}^{2} \Phi & =\frac{-g L^{3} \alpha}{z^{3} \sqrt{f}}\left(\bar{\Psi}_{+} i \gamma^{0} \Psi_{+}+\bar{\Psi}_{-} i \gamma^{0} \Psi_{-}\right) \\
\left(\partial_{z}+\mathcal{A}_{ \pm}\right) \Psi_{ \pm} & =\mp \mathcal{H} \Psi_{\mp}
\end{aligned}
$$

with

$$
\begin{aligned}
\mathcal{A}_{ \pm} & =-\frac{1}{2 z}\left(3-\frac{z f^{\prime}}{2 f}\right) \pm \frac{m L}{z \sqrt{f}} \\
\mathcal{F} & =\frac{i(-\omega+g \Phi)}{\alpha f} \gamma^{0}+\frac{i}{\alpha \sqrt{f}} k_{i} \gamma^{i}
\end{aligned}
$$

as before.

The difficult part is to "impose" staticity and rotational invariance for the non-invariant spinor. This can be done by rephrasing the dynamics in terms of fermion current bilinears, rather than the fermions themselves. We shall first do so rather heuristically, and then show that the equations obtained this way are in fact the flow equations for the Green's functions and composites $\mathcal{J}^{I}(z), G^{I}(z)$ constructed in the previous section. In terms of the local vector currents ${ }^{6}$

$$
J_{+}^{\mu}(x, z)=\bar{\Psi}_{+}(x, z) i \gamma^{\mu} \Psi_{+}(x, z), \quad J_{-}^{\mu}(x, z)=\bar{\Psi}_{-}(x, z) i \gamma^{\mu} \Psi_{-}(x, z),
$$

or equivalently

$J_{+}^{\mu}(p, z)=\int d^{3} k \bar{\Psi}_{+}(-k, z) i \gamma^{\mu} \Psi_{+}(p+k, z), \quad J_{-}^{\mu}(p, z)=\int d^{3} k \bar{\Psi}_{-}(-k, z) i \gamma^{\mu} \Psi_{-}(p+k, z)$.

rotational invariance means that spatial components $J_{ \pm}^{i}$ should vanish on the solution - this solves the constraint from the $A_{i}$ equation of motion, and the equations can be rewritten in terms of $J_{ \pm}^{0}$ only. Staticity and rotational invariance in addition demand that the bilinear momentum $p_{\mu}$ vanish. In other words, we are only considering temporally and spatially averaged densities: $J_{ \pm}^{\mu}(z)=\int d t d^{2} x \bar{\Psi}(t, x, z) i \gamma^{\mu} \Psi(t, x, z)$. Analogous to the bilinear flow equations for the Green's function, we can act with the Dirac operator on the currents to obtain an effective equation of motion, and this averaging over the relative frequencies $\omega$ and momenta $k_{i}$ will set all terms with explicit $k_{i}$-dependence to zero. ${ }^{7}$

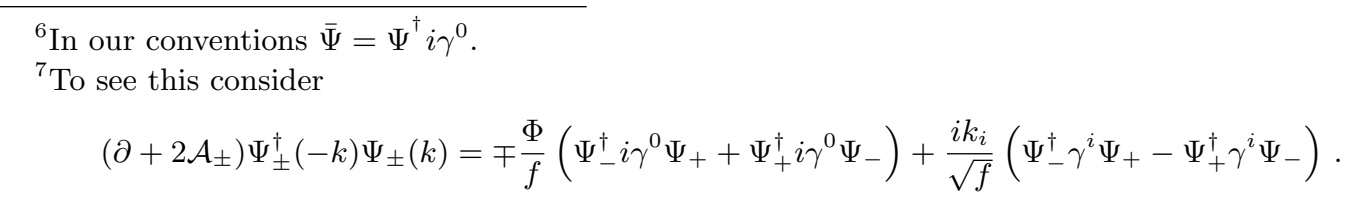

The term proportional to $\Phi$ is relevant for the solution. The dynamics of the term proportional to $k_{i}$ is

$$
\left(\partial+\mathcal{A}_{+}+\mathcal{A}_{-}\right)\left(\Psi_{-}^{\dagger} \gamma^{i} \Psi_{+}-\Psi_{+}^{\dagger} \gamma^{i} \Psi_{-}\right)=-2 i \frac{k^{i}}{\sqrt{f}}\left(\Psi_{+}^{\dagger} \gamma^{0} \Psi_{+}+\Psi_{-}^{\dagger} \gamma^{0} \Psi_{-}\right)
$$

The integral of the r.h.s. over $k^{i}$ vanishes by the assumption of translational and rotational invariance. Therefore the l.h.s. of (3.7) and thus the second term in eq. (3.6) does so as well. 
Restricting to such averaged currents and absorbing a factor of $g / \alpha$ in $\Phi$ and a factor of $g \sqrt{L^{3}}$ in $\Psi_{ \pm}$, we obtain effective equations of motion for the bilinears directly

$$
\begin{aligned}
\left(\partial_{z}+2 \mathcal{A}_{ \pm}\right) J_{ \pm}^{0} & =\mp \frac{\Phi}{f} I \\
\left(\partial_{z}+\mathcal{A}_{+}+\mathcal{A}_{-}\right) I & =\frac{2 \Phi}{f}\left(J_{+}^{0}-J_{-}^{0}\right) \\
\partial_{z}^{2} \Phi & =-\frac{1}{z^{3} \sqrt{f}}\left(J_{+}^{0}+J_{-}^{0}\right),
\end{aligned}
$$

with $I=\bar{\Psi}_{-} \Psi_{+}+\bar{\Psi}_{+} \Psi_{-}$, and all fields are real. The remaining constraint from the $A_{z}$ equation of motion decouples. It demands $\operatorname{Im}\left(\bar{\Psi}_{+} \Psi_{-}\right)=\frac{i}{2}\left(\bar{\Psi}_{-} \Psi_{+}-\bar{\Psi}_{+} \Psi_{-}\right)=0$. What the equations (3.8) tell us is that for nonzero $J_{ \pm}^{0}$ there is a charged electrostatic source for the vector potential $\Phi$ in the bulk.

Momentarily we will motivate the effective equations (3.8) at a more fundamental level. Before that there are several remarks to be made

- These equations contain more information than just current conservation $\partial_{\mu} J^{\mu}=0$. In an isotropic and static background current conservation is trivially true as $\partial_{\mu} J^{\mu}=$ $\partial_{0} J^{0}=-i \int d \omega e^{-i \omega t} \omega J^{0}(\omega)=0$ as $J^{0}(\omega \neq 0)=0$.

- We have scaled out the electromagnetic coupling. $\mathrm{AdS}_{4} / \mathrm{CFT}_{3}$ duals for which the underlying string theory is known generically have $g=\kappa / L$ with $\kappa$ the gravitational coupling constant as defined in (2.3). Thus, using standard $\mathrm{AdS}_{4} / \mathrm{CFT}_{3}$ scaling, a finite charge in the new units translates to a macroscopic original charge of order $L / \kappa \propto N^{1 / 3}$. This large charge demands that backreaction of the fermions in terms of its bilinear is taken into account as a source for $\Phi$.

- The equations are local. From the fundamental point of view, that one considers finite density in the bulk, this is strange to say the least. Generic multi-fermion configurations are non-local, see e.g. [38]. These equations can therefore never capture the full bulk fermion dynamics. Our starting point has been a single fermion perspective, where the Pauli blocking induced non-locality is absent. In that context local equations are fine. We have also explicitly averaged over all directions parallel to the boundary and, as we have shown in the previous section (see also footnote 7), it is this averaging that tremendously simplifies the equations. The most curious part may be that this unaveraged set of equations - and therefore also eqs (3.8) - are all local in the radial direction $z$. From the AdS perspective a many-fermion system should be non-local democratically and thus also exhibit non-locality in $z$, yet from the CFT perspective where $z$-dynamics encode RG-flow, it is eminently natural. We leave the resolution of this paradox to future work.

The justification of using (3.8) to construct the AdS dual of a regular Fermi liquid is the connection between local fermion bilinears and the CFT Green's function. The complicated flow equations (2.29) reduce precisely to the first two equations in (3.8) upon performing the spacetime averaging and the trace, i.e. $J_{ \pm}^{0}=\int d^{3} k \operatorname{Tr} \mathcal{J}_{ \pm}^{0}$ and $I=\int d^{3} k \operatorname{Tr}\left(G^{\mathbb{1}}+\bar{G}^{\mathbb{1}}\right)$. 
Combined with the demand that we only consider normalizable solutions and the proof that $\mathcal{J}_{-}^{0}$ is proportional to the pole-strength, the radial evolution equations (3.8) are the (complicated) AdS recasting of the RG-flow for the pole-strength. This novel interpretation ought to dispel some of the a priori worries about our unconventional treatment of the fermions through their semi-classical bilinears. There is also support from the gravity side, however. Recall that for conventional many-body systems and fermions in particular one first populates a certain set of states and then tries to compute the macroscopic properties of the collective. In a certain sense the equations (3.8) formulate the same program but in opposite order: one computes the generic wavefunction charge density with and by imposing the right boundary conditions, i.e normalizability, one selects only the correct set of states. This follows directly from the equivalence between normalizable AdS modes and quasiparticle poles that are characterized by well defined distinct momenta $k_{F}$ (for $\left.\omega=\omega_{F} \equiv 0\right)$. The demand that any non-trivial Dirac hair black hole is constructed from normalizable solutions of the composite operators (i.e. their leading and subleading asymptotes vanish ${ }^{8}$ ) thus means that one imposes a superselection rule on the spatial averaging in the definition of $J_{ \pm}^{I}$ :

$$
\begin{aligned}
\left.J_{ \pm}^{0}(z)\right|_{\text {normalizable }} & \left.\equiv \int d^{3} k \bar{\Psi}_{ \pm}(-k) i \gamma^{0} \Psi_{ \pm}(k)\right|_{\text {normalizable }} \\
& =\int d^{3} k \delta^{2}\left(|k|-\left|k_{F}\right|\right)\left|B_{ \pm}^{(0)}(k)\right|^{2} z^{4+2 m \pm 1}+\ldots
\end{aligned}
$$

We see that the constraint of normalizability from the bulk point of the view, implies that one selects precisely the on-shell bulk fermion modes as the building blocks of the density $J_{ \pm}^{0}$.

In turn this means that the true system that eqs. (3.8) describe is somewhat obscured by the spatial averaging. Clearly even a single fermion wavefunction is in truth the full set of two-dimensional wavefunctions whose momentum $k^{i}$ has length $k_{F}$. However, the averaging could just as well be counting more, as long as there is another set of normalizable states once the isotropic momentum surface $|k|=\left|k_{F}\right|$ is filled. Pushing this thought to the extreme, one could even speculate that the system (3.8) gives the correct quantummechanical description of the many-body Fermi system: the system which gravitational reasoning suggests is the true ground state of the charged AdS black hole in the presence of fermions.

To remind us of the ambiguity introduced by spatial averaging, we shall give the boundary coefficient of normalizable solution for $J_{-}^{0}=\int d^{3} k \mathcal{J}_{-}^{0}$ a separate name. The quantity $\mathcal{J}_{-}^{0}\left(z_{0}\right)$ is proportional to the pole strength, which via Migdal's relation quantifies the characteristic occupation number discontinuity at $\omega_{F} \equiv 0$. We shall therefore call the coefficient $\left.\int d^{3} k\left|B_{-}\right|^{2}\right|_{\text {normalizable }}=\Delta n_{F}$.

\footnotetext{
${ }^{8}$ One can verify that the discussion in section 2.1 .1 holds also for fully backreacted solutions. The derivation there builds on the assumption that the boundary behavior of the electrostatic potential is regular. It is straightforward to check in (3.8) that indeed precisely for normalizable solutions, i.e. in the absence of explicit fermion-sources, when both the leading and subleading terms in $J_{ \pm}^{0}$ and $I$ vanish, the boundary behavior the scalar potential remains regular, as required.
} 


\subsection{Thermodynamics}

At a very qualitative level the identification $\left.J_{-}^{0}\right|_{\text {norm }}(z) \equiv \Delta n_{F} z^{3+2 m}+\ldots$ can be argued to follow from thermodynamics as well. From the free energy for an AdS dual solution to a Fermi liquid, one finds that the charge density directly due to the fermions is

$$
\rho_{\text {total }}=-2 \frac{\partial}{\partial \mu} F=\frac{-3}{2 m+1} \frac{\Delta n_{F}}{z_{0}^{-1-2 m}}+\rho+\ldots,
$$

with $z_{0}^{-1}$ the UV-cutoff as before. The cut-off dependence is a consequence of the fact that the system is interacting, and one cannot truly separate out the fermions as free particles. Were one to substitute the naive free fermion scaling dimension $\Delta=m+3 / 2=1$, the cutoff dependence would vanish and the identification would be exact.

We can thus state that in the interacting system there is a contribution to the charge density from a finite number of fermions proportional to

$$
\rho_{F}=\frac{-3}{2 \Delta-2} \frac{\Delta n_{F}}{z_{0}^{2-2 \Delta}}+\ldots,
$$

although this contribution formally vanishes in the limit where we send the UV-cutoff $z_{0}^{-1}$ to infinity.

To derive eq. (3.10), recall that the free energy is equal to minus the on-shell action of the AdS dual theory. Since we disregard the gravitational backreaction, the Einstein term in the AdS theory will not contain any relevant information and we consider the Maxwell and Dirac term only. We write the action as,

$$
S=\int_{z_{0}}^{1} \sqrt{-g}\left[\frac{1}{2} A_{N} D_{M} F^{M N}-\bar{\Psi} \not D \Psi-m \bar{\Psi} \Psi\right]+\oint_{z=z_{0}} \sqrt{-h}\left(\bar{\Psi}_{+} \Psi_{-}+\frac{1}{2} A_{\mu} n_{\alpha} F^{\alpha \mu}\right),
$$

where we have included an explicit fermionic boundary term that follows from the AdS/CFT dictionary [12] and $n_{\alpha}$ is a normal vector to the boundary. The boundary action is not manifestly real, but its on-shell value which contributes to the free energy is real. Recall that the imaginary part of $\bar{\Psi}_{+} \Psi_{-}$decouples from eqs. (3.8). The boundary Dirac term in (3.12) is therefore equal to $I=2 \operatorname{Re}\left(\bar{\Psi}_{+} \Psi_{-}\right)$.

To write the free energy in terms of the quantities $\mu, \rho$ and $\Delta n_{F}$, note that the on-shell bulk Dirac action vanishes. Importantly the bulk Maxwell action does contribute to the free energy. Its contribution is

$$
\begin{aligned}
F_{\text {bulk }} & =\lim _{z_{0} \rightarrow 0} \int_{z_{0}}^{1} d z d^{3} x\left[\frac{1}{2} \Phi \partial_{z z} \Phi\right]_{\text {on-shell }} \\
& =-\lim _{z_{0} \rightarrow 0} \int_{z_{0}}^{1} d z d^{3} x\left[\frac{1}{2 z^{3} \sqrt{f}} \Phi\left(J_{+}^{0}+J_{-}^{0}\right)\right]_{\text {on-shell }},
\end{aligned}
$$

where we have used the equation of motion (3.8). This contribution should be expected, since the free energy should be dominated by infrared, i.e. near horizon physics. Due to the logarithmic singularity in the electrostatic potential (eq. (3.17) this bulk contribution diverges, but this divergence should be compensated by gravitational backreaction. At the same time the singularity is so mild, however, that the free energy, the integral of the Maxwell term, remains finite in the absence of the Einstein contribution. 
Formally, i.e. in the limit $z_{0} \rightarrow 0$, the full free energy arises from this bulk contribution (3.13). The relation (3.10) between the charge density and $\Delta n_{F}$ follows only from the regularized free energy, and is therefore only a qualitative guideline. Empirically, as we will show, it is however, a very good one (see figure 1 in the next section). Splitting the regularized bulk integral in two

$F_{\text {bulk }}=\int_{z^{*}}^{1} d z d^{3} x\left[\frac{1}{2 z^{3} \sqrt{f}} \Phi\left(J_{+}^{0}+J_{-}^{0}\right)\right]_{\text {on-shell }}+\lim _{z_{0} \rightarrow 0} \int_{z_{0}}^{z_{*}} d z d^{3} x\left[\frac{1}{2 z^{3} \sqrt{f}} \Phi\left(J_{+}^{0}+J_{-}^{0}\right)\right]_{\text {on }- \text { shell }}$,

we substitute the normalizable boundary behavior of $\Psi_{+}=B_{+} z^{5 / 2+m}+\ldots, \Psi_{-}=$ $B_{-} z^{3 / 2+m}+\ldots$ and $\Phi=\mu-\rho z+\ldots$, and obtain for the regularized free energy

$F=F_{\text {horizon }}\left(z_{*}\right)+\lim _{z_{0} \rightarrow 0} \int_{z_{0}}^{z_{*}} d^{3} x d z\left[\frac{-1}{2 z^{3}} \mu\left|B_{-}\right|^{2} z^{3+2 m}+\ldots\right]+\oint \frac{d^{3} x}{z_{0}^{3}}\left[-\bar{B}_{+} B_{-} z_{0}^{4+2 m}+\frac{1}{2} \mu \rho z_{0}^{3}\right]$.

Using that $B_{+}=-i \mu \gamma^{0} B_{-} /(2 m+1)$ (eq. (2.10)), the second bulk term and boundary contribution are proportional, and the free energy schematically equals

$$
F=F^{\text {horizon }}+\lim _{z_{0} \rightarrow 0} \int d^{3} x\left[\frac{3 \mu}{2(2 m+1)} \bar{B}_{-} i \gamma^{0} B_{-} z_{0}^{1+2 m}-\frac{1}{2} \mu \rho\right] .
$$

With the UV-regulator $z_{0}^{-1}$ finite, this yields the charge density in eq. (3.10) after one recalls that $\bar{B}_{-}=B_{-}^{\dagger} i \gamma^{0}$.

With the derived rule that the AdS dual to a Fermi liquid has a nonzero normalizable component in the current $J_{-}^{0}$, we will now construct an AdS solution that has this property: an AdS black hole with Dirac hair. Ignoring backreaction, these are solutions to the density equations (3.8). In its simplest form the interpretation is that of the backreaction due to a single fermion wavefunction, but as explained the spatial averaging of the density combined with the selection rule of normalizability could be capturing a more general solution.

\subsection{At the horizon: entropy collapse to a Lifshitz solution}

Before we can proceed with the construction of non-trivial Dirac hair solutions to eqs. (3.8), we must consider the boundary conditions at the horizon necessary to solve the system. Insisting that the right-hand-side of the dynamical equations (3.8) is subleading at the horizon, the near-horizon behavior of $J_{ \pm}^{0}, I, \Phi$ is:

$$
\begin{aligned}
J_{ \pm}^{0} & =J_{\text {hor }, \pm}(1-z)^{-1 / 2}+\ldots, \\
I & =I_{\text {hor }}(1-z)^{-1 / 2}+\ldots, \\
\Phi & =\Phi_{\text {hor }}^{(1)}(1-z) \ln (1-z)+\left(\Phi_{\text {hor }}^{(2)}-\Phi_{\text {hor }}^{(1)}\right)(1-z)+\ldots
\end{aligned}
$$

If we insist that $\Phi$ is regular at the horizon $z=1$, i.e. $\Phi_{\text {hor }}^{(1)}=0$, so that the electric field is finite, the leading term in $J_{ \pm}^{0}$ must vanish as well, i.e. $J_{\text {hor }, \pm}=0$, and the system reduces to a free Maxwell field in the presence of an AdS black hole and there is no fermion density profile in the bulk. Thus in order to achieve a nonzero fermion profile in the bulk, we must have an explicit source for the electric-field on the horizon. Strictly speaking, this 
invalidates our neglect of backreaction as the electric field and its energy density at the location of the source will be infinite. As we argued above, this backreaction is in fact expected to resolve the finite ground-state entropy problem associated with the presence of a horizon. The backreaction should remove the horizon completely, and the background should resemble the horizonless metrics found in [26, 27, 39]; the same horizon logarithmic behavior in the electrostatic potential was noted there. Nevertheless, as the divergence in the electric field only increases logarithmically as we approach the horizon, and our results shall hinge on the properties of the equations at the opposite end near the boundary, we shall continue to ignore it here. We shall take the sensibility of our result after the fact, as proof that the logarithmic divergence at the horizon is indeed mild enough to be ignored.

The identification of the boundary value of $J_{-}^{0}$ with the Fermi liquid characteristic occupation number jump $\Delta n_{F}$ rested on the insistence that the currents are built out of AdS Dirac fields. This deconstruction also determines a relation between the horizon boundary conditions of the composite fields $J_{ \pm}^{0}$, I. If $\Psi_{ \pm}(z)=C_{ \pm}(1-z)^{-1 / 4}+\ldots$ then $J_{\text {hor }, \pm}=C_{ \pm}^{2}$ and $I_{\text {hor }}=C_{+} C_{-}$. As the solution $\Phi_{\text {hor }}^{(1)}$ is independent of the solution $\Phi_{\text {hor }}^{(2)}$ which is regular at the horizon, we match the latter to the vector-potential of the charged AdS black hole: $\Phi_{\text {hor }}^{(2)}=-2 g q \equiv g \mu_{0} / \alpha$. Recalling that $\Phi_{\text {hor }}^{(1)}=-\left(J_{\text {hor, }+}+J_{\text {hor, }-}\right)$, we see that the three-parameter family of solutions at the horizon in terms of $C_{ \pm}, \Phi_{\text {hor }}^{(2)}$ corresponds to the three-parameter space of boundary values $A_{+}, B_{-}$and $\mu$ encoding a fermion-source, the fermion-response/expectation value and the chemical potential.

We can now search whether within this three-parameter family a finite normalizable fermion density solution with vanishing source $A_{+}=0$ exists for a given temperature $T$ of the black hole.

\subsection{A BH with Dirac hair}

The equations are readily solved numerically with a shooting method from the horizon. We consider both an uncharged AdS-Schwarzschild solution and the charged AdS ReissnerNordström solution. Studies of bosonic condensates in AdS/CFT without backreaction have mostly been done in the AdS-Schwarzschild (AdSS) background ([19, 20] and references therein). An exception is [40], which also considers the charged RN black hole. As is explained in [40], they correspond to two different limits of the exact solution: the AdSS case requires that $\Delta n_{F} \gtrsim \mu$ that is, the total charge of the matter fields should be dominant compared to the charge of the black hole. On the other hand, the RN limit is appropriate if $\Delta n_{F} \ll \mu$. It ignores the effect of the energy density of the charged matter sector on the charged black hole geometry. The AdS Schwarzschild background is only reliable near $T_{c}$, as at low temperatures the finite charged fermion density is comparable to $\mu$. The RN case is under better control for low temperatures, because near $T=0$ the chemical potential can be tuned to stay larger than fermion density.

We shall therefore focus primarily on the solution in the background of an AdS RN black hole, i.e. the system with a heat bath with chemical potential $\mu$ - non-linearly determined by the value of $\Phi_{\text {hor }}^{(2)}=\mu_{0}$ at the horizon — which for low $T / \mu$ should show the characteristic $\Delta n_{F}$ of a Fermi liquid. The limit in which we may confidently ignore backreaction is $\Phi_{\text {hor }}^{(1)} \ll \mu_{0}$ for $T \lesssim \mu_{0}$ - for AdSS the appropriate limit is $\Phi_{\text {hor }}^{(1)} \ll T$ for $\mu_{0} \ll T$. 


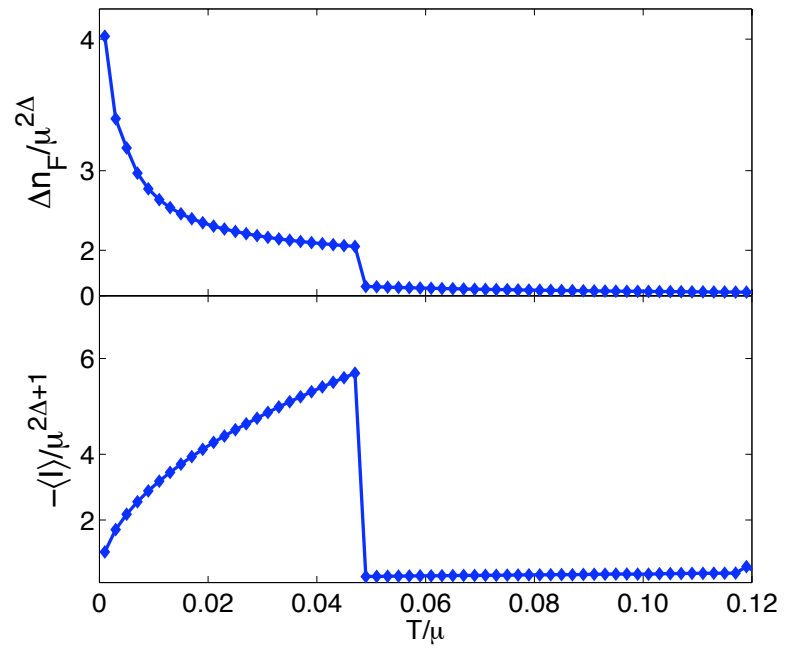

(a)

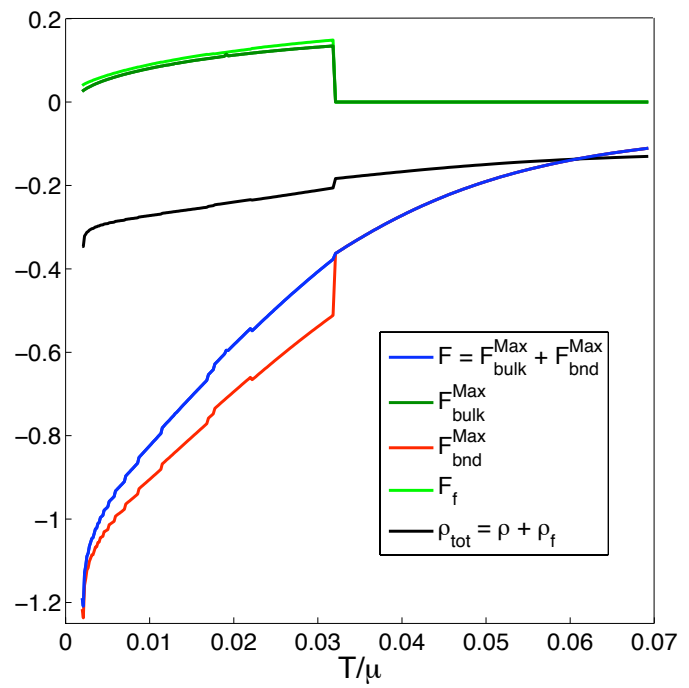

(b)

Figure 1. (a) Temperature dependence of the Fermi liquid occupation number discontinuity $\Delta n_{F}$ and operator $I$ for a fermionic field of mass $m=-1 / 4$ dual to an operator of dimension $\Delta=5 / 4$. We see a large density for $T / \mu$ small and discontinuously drop to zero at $T \approx 0.05 \mu$. At this same temperature, the proxy free energy contribution per particle (the negative of $I$ ) vanishes. (b) The free energy $F=F^{\text {fermion }}+F^{\text {Maxwell }}$ (eq. (3.12)) as a function of $T / \mu$ ignoring the contribution from the gravitational sector. The blue curve shows the total free energy $F=F^{\text {Maxwell }}$, which is the sum of a bulk and a boundary term. The explicit fermion contribution $F_{\text {fermion }}$ vanishes, but the effect of a non-zero fermion density is directly encoded in a non-zero $F_{b u l k}^{\mathrm{Maxwell}}$. The figure also shows this bulk $F_{\text {bulk }}^{\text {Maxwell }}$ and the boundary contribution $F_{\text {bulk }}^{\text {Maxwell }}$ separately and how they sum to a continuous $F_{\text {total }}$. Although formally the explicit fermion contribution $F_{f} \sim I$ in equation (3.16) vanishes, the bulk Maxwell contribution is captured remarkably well by its value when the cut-off is kept finite. The light-green curve in the figure shows $F_{f}$ for a finite $z_{0} \sim 10^{-6}$. For completeness we also show the total charge density, eq. (3.10). The dimension of the fermionic operator used in this figure is $\Delta=1.1$.

\subsubsection{Finite fermion density solutions in AdS-RN}

Figure 1 shows the behavior of the occupation number discontinuity $n_{F} \equiv\left|B_{-}\right|^{2}$ and the fermion free-energy contribution $I$ as a function of temperature in a search for normalizable solutions to eqs.(3.8) with the aforementioned boundary conditions. We clearly see a first order transition to a finite fermion density, as expected. The underlying Dirac field dynamics can be recognized in that the normalizable solution for $J_{-}^{0}(z)$ which has no leading component near the boundary by construction, also has its subleading component vanishing (figure 2). ${ }^{9}$

\footnotetext{
${ }^{9}$ Although the Dirac hair solution has charged matter in the bulk, there is no Higgs effect for the bulk gauge field, and thus there is no direct spontaneous symmetry breaking in the boundary. Indeed one would not expect it for the Fermi liquid ground state. There will be indirect effect on the conductivity similar to [27]. We thank Andy O'Bannon for his persistent inquiries to this point.
} 


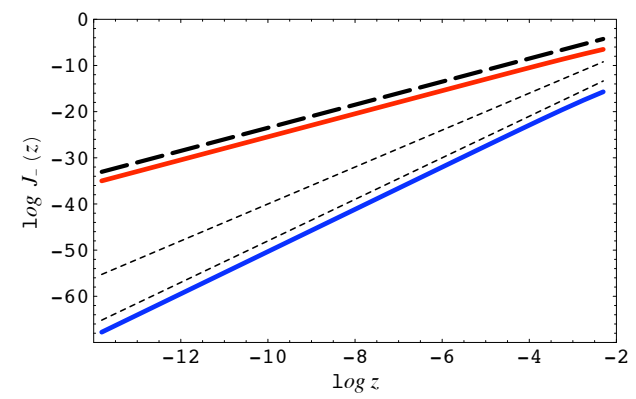

Figure 2. The boundary behaviour of $J_{-}(0)$ in for a generic solution (blue) to eqs. (3.8) and a normalizable Dirac-hair solution (red) for $m=-1 / 4$ in the background of an AdS-RN black hole with $\mu / T=128.8$. The dotted lines show the scaling $z^{11 / 2}$ and $z^{4}$ of the leading and subleading terms in an expansion of $J_{-}^{0}(z)$ near $z=0$; the dashed line shows the scaling $z^{5 / 2}$ of the subsubleading expansion whose coefficient is $\left|B_{-}\left(\omega_{F}, k_{F}\right)\right|^{2}$. That the Dirac hair solution (red) scales as the subsubleading solution indicates that the current $J_{-}^{0}$ faithfully captures the density of the underlying normalizable Dirac field.

Analyzing the transition in more detail in figure 3, we find:

1. The dimensionless number discontinuity $\Delta n_{F} / \mu^{2 \Delta}$ scales as $T^{-\delta}$ in a certain temperature range $T_{F}<T<T_{c}$, with $\delta>0$ depending on $g$ and $\Delta$, and $T_{F}$ typically very small. At $T=T_{c}>T_{F}$ it drops to zero discontinuously, characteristic of a first order phase transition.

2. At low temperatures, $0<T<T_{F}$, the power-law growth comes to a halt and ends with a plateau where $\Delta n_{F} / \mu^{2 \Delta} \sim$ const. (figure 3a). It is natural to interpret this temperature as the Fermi temperature of the boundary Fermi liquid.

3. The fermion free energy contribution $I / \mu^{2 \Delta+1}$ scales as $T^{1 / \nu}$ with $\nu>1$ for $0<T<$ $T_{c}$, and drops to zero discontinuously at $T_{c}$. As $I$ empirically equals minus the free energy per particle, it is natural that $I(T=0)=0$, and this in turn supports the identification of $\Delta n_{F}(T=0)$ as the step in number density at the Fermi energy.

One expects that the exponents $\delta, \nu$ are controlled by the conformal dimension $\Delta \cdot{ }^{10}$ The dependence of the exponent $\delta$ on the conformal dimension is shown in figure 3a. While a correlation clearly exists, the data are not conclusive enough to determine the relation $\delta=\delta(\Delta)$. The clean power law $T^{-\delta}$ scaling regime is actually somewhat puzzling. These values of the temperature, $T_{F}<T<T_{c}$, correspond to a crossover between the true Fermi liquid regime for $T<T_{F}$ and the conformal phase for $T>T_{c}$, hence there is no clear ground for a universal scaling relation for $\delta$, which seems to be corroborated by the data (figure $3 \mathrm{~b}$ ). At the same time, the scaling exponent $\nu$ appears to obey $\nu=2$ with great precision (figure $3 \mathrm{~b}$, inset) independent of $\Delta$ and $g$.

A final consideration, needed to verify the existence of a finite fermion density AdS solution dual to a Fermi liquid, is to show that the ignored backreaction stays small. In particular, the divergence of the electric field at the horizon should not affect the result.

\footnotetext{
${ }^{10}$ The charge $g$ of the underlying conformal fermionic operator scales out of the solution.
} 


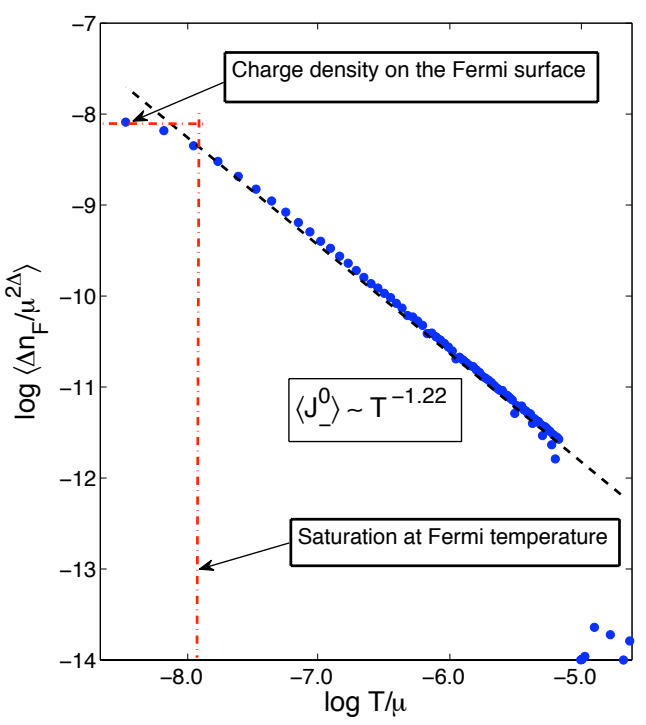

(a)

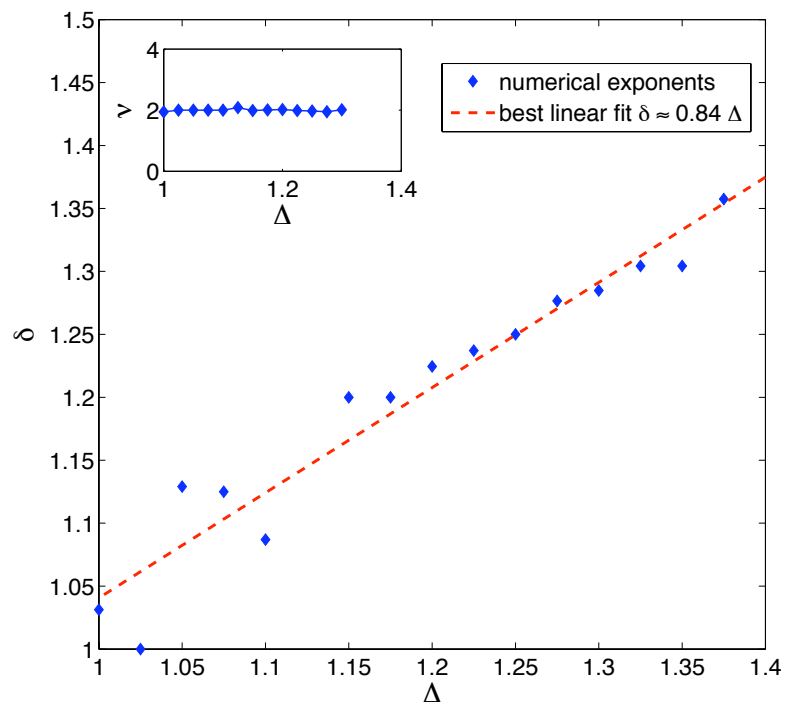

(b)

Figure 3. (a) Approximate power-law scaling of the Fermi liquid characteristic occupation number discontinuity $\Delta n_{F} / \mu^{2 \Delta} \sim T^{-\delta}$ as a function of $T / \mu$ for $\Delta=5 / 4$. This figure clearly shows the saturation of the density at very low $T / \mu$. The saturation effect is naturally interpreted as the influence of the characteristic Fermi energy. (b) The scaling exponent $\delta$ for different values of the conformal dimension $\Delta$. There is a clear correlation, but the precise relation cannot be determined numerically. The scaling exponent of the current $I / \mu^{2 \Delta+1} \sim T^{-1 / \nu}$ obeys $\nu=2$ with great accuracy, on the other hand (Inset).

The total bulk electric field $E_{z}=-\partial_{z} \Phi$ is shown in figure 4 a, normalized by its value at $z=1 / 2$. The logarithmic singularity at the horizon is clearly visible. At the same time, the contribution to the total electric field from the charged fermions is negligible even very close to the horizon. ${ }^{11}$ This suggests that our results are robust with respect to the details of the IR divergence of the electric field.

The diverging backreaction at the horizon is in fact the gravity interpretation of the first order transition at $T_{c}$ : an arbitrarily small non-zero density leads to an abrupt change in the on shell bulk action. As the latter is the free energy in the CFT, it must reflect the discontinuity of a first order transition. A full account of the singular behavior at the horizon requires self-consistent treatment including the Einstein equations. At this level, we can conclude that the divergent energy density at the horizon implies that the near-horizon physics becomes substantially different from the $\mathrm{AdS}_{2}$ limit of the $\mathrm{RN}$ metric. It is natural to guess that the RN horizon disappears completely, corresponding to a ground state with zero entropy, as hypothesized in [26]. This matches the expectation that the finite fermidensity solution in the bulk describes the Fermi-liquid. The underlying assumption in the above reasoning is that the total charge is conserved.

\footnotetext{
${ }^{11}$ It is of the order $10^{-4}$, starting from $z=0.9999$. We have run our numerics using values between $1-10^{-6}$ and $1-10^{-2}$ and found no detectable difference in quantities at the boundary.
} 

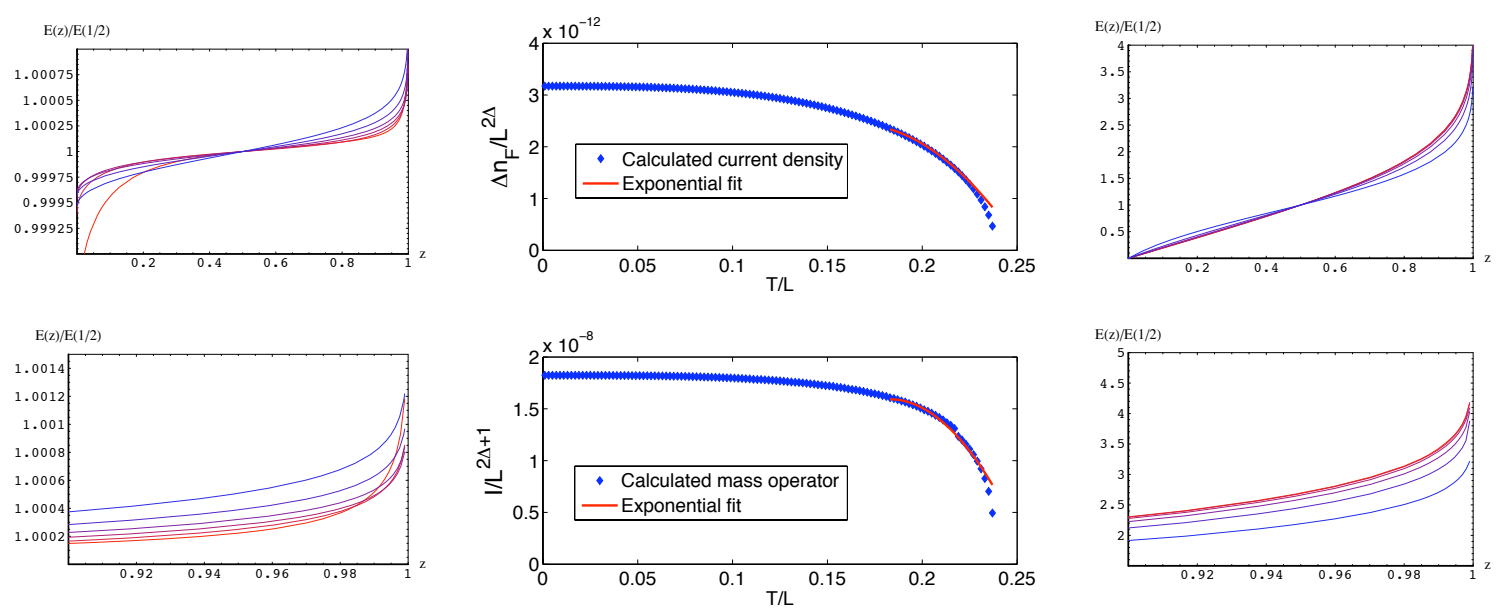

(a)

(b)

(c)

Figure 4. (a) The radial electric field $-E_{z}=\partial \Phi / \partial z$, normalized to the midpoint value $E_{z}(z) / E_{z}(1 / 2)$ for whole interior of the finite fermion density AdS-RN solution (upper) and near the horizon (lower). One clearly sees the soft, log-singularity at the horizon. The colors correspond to increasing temperatures from $T=0.04 \mu$ (lighter) to $T=0.18 \mu$ (darker), all with $\Delta=1.1$. (b) The occupation number jump $\Delta n_{F}$ and free energy contribution $I$ as a function of temperature in AdS-Schwarzschild. We see the jump $\Delta n_{F}$ saturate at low temperatures and fall off at high $T$. An exponential fit to the data (red curve) shows that in the critical region the fall-off is stronger than exponential, indicating that the transition is first order. The conformal dimension of the fermionic operator is $\Delta=1.1$. (c) The radial electric field $-E_{z}=\partial \Phi / \partial z$, normalized to the midpoint value $\left(E_{z}(z) / E_{z}(1 / 2)\right)$ for the finite fermion density AdS-Schwarzschild background. The divergence of the electric field $E_{z}$ is again only noticeable near the horizon and can be neglected in most of the bulk region.

\subsubsection{Finite fermion density in AdSS}

For completeness, we will describe the finite fermion-density solutions in the AdS Schwarzschild geometry as well. In these solutions the charge density is set by the density of fermions alone. They are therefore not reliable at very low temperatures $T \ll T_{c}$ when gravitational backreaction becomes important. The purpose of this section is to show the existence of finite density solutions does not depend on the presence of a charged black hole set by the horizon value $\Phi_{\text {hor }}^{(2)}=\mu_{0}$, but that the transition to a finite fermion density can be driven by the charged fermions themselves.

Figure 4b shows the nearly instantaneous development of a non-vanishing expectation value for the occupation number discontinuity $\Delta n_{F}$ and $I$ in the AdS Schwarzschild background. The rise is not as sharp as in the RN background. It is, however, steeper than exponential, and we may conclude that the system undergoes a discontinuous first order transition to a AdS Dirac hair solution. The constant limit reached by the fermion density as $T \rightarrow 0$ has no meaning as we cannot trust the solution far away from $T_{c}$.

The backreaction due to the electric field divergence at the horizon can be neglected, for the same reason as before (figure 4c). 


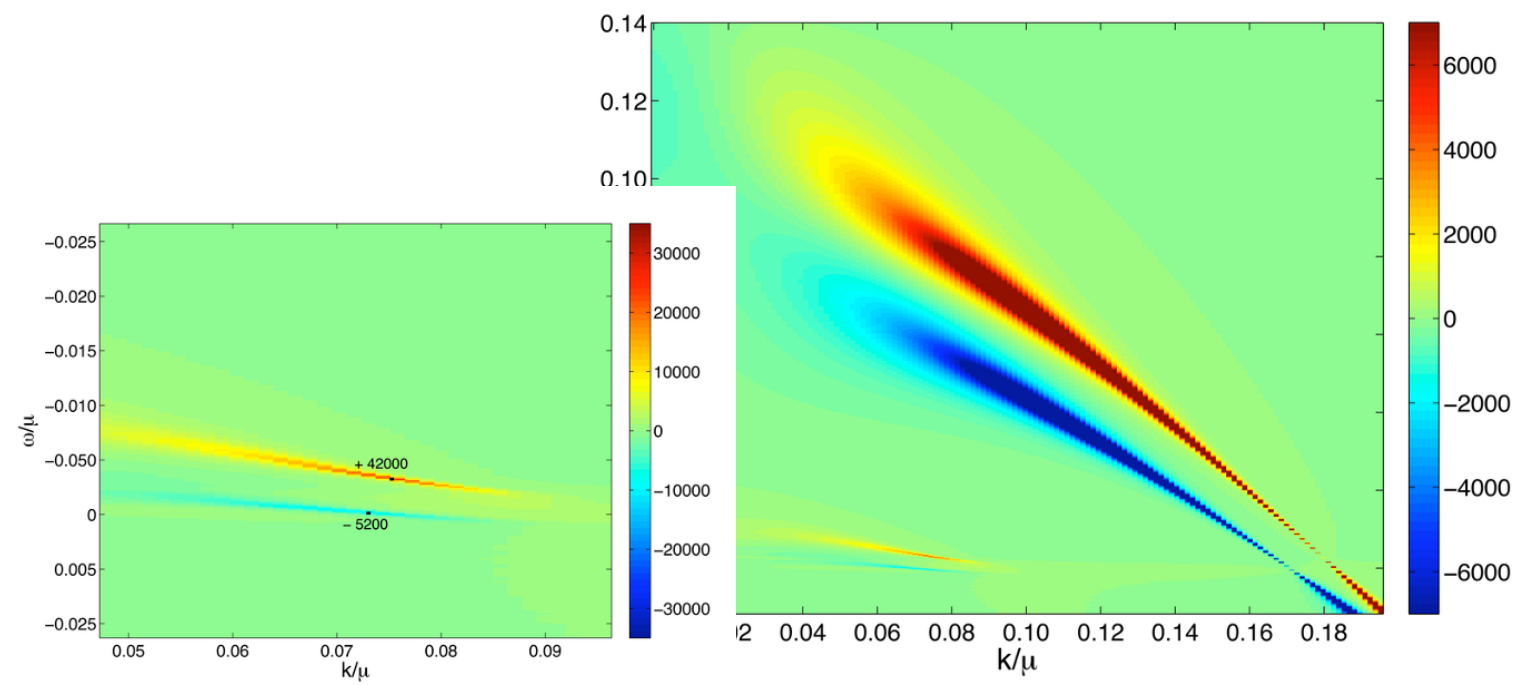

Figure 5. The single-fermion spectral function in the probe limit of pure AdS Reissner-Nordström (red/yellow) minus the spectrum in the finite density system (blue). The conformal dimension is $\Delta=5 / 4$, the probe charge $g=2$, and $\mu / T=135$. We can see two quasiparticle poles near $\omega=0$, a non-FL pole with $k_{F}^{\text {probe }} \simeq 0.11 \mu$ and $k_{F}^{\Delta n_{F}} \simeq 0.08 \mu$ respectively and a FL-pole with $k_{F}^{\text {probe }} \simeq 0.18 \mu$ and $k_{F}^{\Delta n_{F}} \simeq 0.17 \mu$. The dispersion of both poles is visibly similar between the probe and the finite density backgroudnd. At the same time, the non-FL pole has about 8 times less weight in the finite density background, whereas the FL-pole has gained about 6.5 times more weight.

\subsection{Confirmation from fermion spectral functions}

If, as we surmised, the finite fermion density phase is the true Fermi-liquid-like ground state, the change in the fermion spectral functions should be minimal as the characteristic quasiparticle peaks are already present in the probe limit, i.e. pure AdS Reissner-Nordström [11, 12]. Figure 5 shows that quasiparticle poles near $\omega=0$ with similar analytic properties can be identified in both the probe pure AdS-RN case and the AdS-RN Dirac-hair solution. The explanation for this similarity is that the electrostatic potential $\Phi$ almost completely determines the spectrum, and the change in $\Phi$ due to the presence of a finite fermion density is quite small. Still, one expects that the finite fermion density system is a more favorable state. This indeed follows from a detailed comparison between the spectral functions $A(\omega ; k)$ in the probe limit and the fermion-liquid phase (figure 5 ). We see that:

1. All quasiparticle poles present in the probe limit are also present in the Dirac hair phase, at a slightly shifted value of $k_{F}$. This shift is a consequence of the change in the bulk electrostatic potential $\Phi$ due to the presence of the charged matter. For a Fermi-liquid-like quasiparticle corresponding to the second pole in the operator with $\Delta=5 / 4$ and $g=2$ we find $k_{F}^{\text {probe }}-k_{F}^{\Delta n_{F}}=0.07 \mu$. The non-Fermi-liquid pole, i.e. the first pole for the same conformal operator, has $k_{F}^{\text {probe }}-k_{F}^{\Delta n_{F}}=0.03 \mu$.

2. The dispersion exponents $\nu$ defined through $\left(\omega-E_{F}\right)^{2} \sim\left(k-k_{F}\right)^{2 / \nu}$, also maintain roughly the same values as both solutions. This is visually evident in the near similar 
slopes of the ridges in figure 5. In the AdS Reissner-Nordström background, the dispersion coefficients are known analytically as a function of the Fermi momentum: $\nu_{k_{F}}=\sqrt{2 \frac{k_{F}^{2}}{\mu^{2}}-\frac{1}{3}+\frac{1}{6}(\Delta-3 / 2)^{2}}$ [13]. The Fermi-liquid-like quasiparticle corresponding to the second pole in the operator with $\Delta=5 / 4$ and $g=2$ has $\nu_{k_{F}}^{\text {probe }}=1.02$ vs. $\nu^{\Delta n_{F}}=1.01$. The non-Fermi-liquid pole corresponding to the first pole for the same conformal operator, has $\nu_{k_{F}}^{\text {probe }} \approx 0.10$, and $\nu^{\Delta n_{F}}=0.12$.

3. The most distinct property of the finite density phase is the redistributed spectral weight of the poles. The non-Fermi liquid pole reaches its maximum height about $10^{4}$, an order of magnitude less than in the probe limit, whereas the second, Fermi liquidlike pole, increases by an order of magnitude. This suggests that the finite density state corresponds to the Fermi-liquid like state, rather than a non-Fermi liquid.

4. As we mentioned in the introduction, part of the reason to suspect the existence of an AdS-RN Dirac-hair solution is that a detailed study of spectral functions in AdS-RN reveals that the quasiparticle peak is anomalously sensitive to changes in $T$. This anomalous temperature dependence disappears in the finite density solution. Specifically in pure AdS-RN the position $\omega_{\max }$ where the peak height is maximum, denoted $E_{F}$ in [12], does not agree with the value $\omega_{\text {pole }}$, where the pole touches the real axis in the complex $\omega$-plane, for any finite value of $T$, and is exponentially sensitive to changes in $T$ (figure 6). In the AdS-RN Dirac hair solution the location $\omega_{\text {max }}$ and the location $\omega_{\text {pole }}$ do become the same. Figure $6 \mathrm{~b}$ shows that the maximum of the quasiparticle peak always sits at $\omega \simeq 0$ in finite density Dirac hair solution, while it only reaches this as $T \rightarrow 0$ in the probe AdS-RN case.

\section{Discussion and conclusion}

Empirically we know that the Fermi liquid phase of real matter systems is remarkably robust and generic. This is corroborated by analyzing effective field theory around the Fermi surface, but as it assumes the ground state it cannot explain its genericity. If the Fermi liquid ground state is so robust, this must also be a feature of the recent holographic approaches to strongly interacting fermionic systems. Our results here indicate that this is so. We have used Migdal's relation to construct AdS/CFT rules for the holographic dual of a Fermi liquid: the characteristic occupation number discontinuity $\Delta n_{F}$ is encoded in the normalizable subsubleading component of the spatially averaged fermion density $J_{-}^{0}(z) \equiv$ $\int d^{3} k \bar{\Psi}(\omega=0,-k, z) i \gamma^{0} \Psi(\omega=0, k, z)$ near the AdS boundary. This density has its own set of evolution equations, based on the underlying Dirac field, and insisting on normalizability automatically selects the on-shell wavefunctions of the underlying Dirac-field.

The simplest AdS solution that has a non-vanishing expectation value for the occupation number discontinuity $\Delta n_{F}$ is that of a single fermion wavefunction. Using the density approach - which through the averaging appears to describe a class of solutions rather than one specific solution - we have constructed the limit of this solution where gravitational backreaction is ignored. At low black hole temperatures this solution with fermionic 

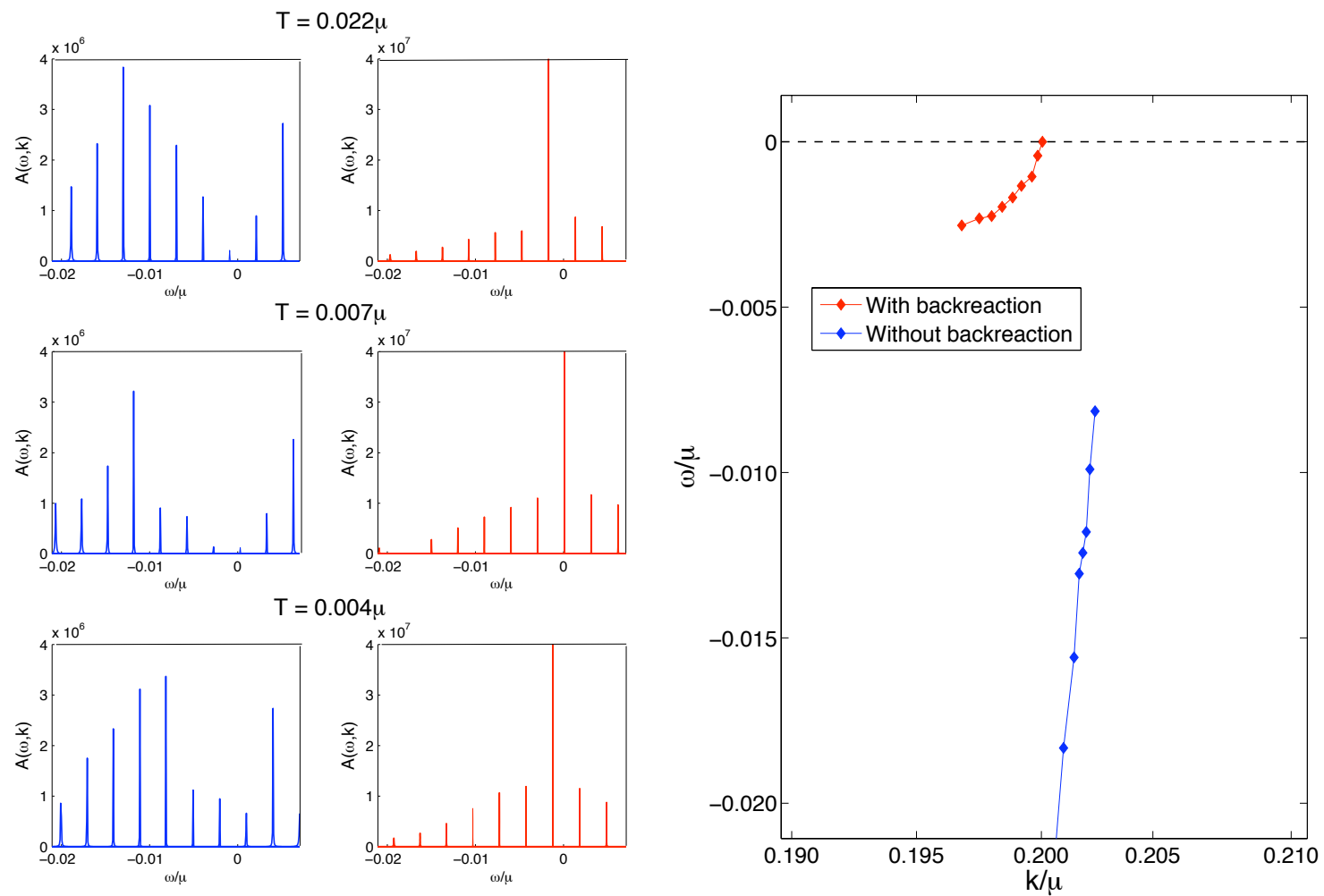

(a)

(b)

Figure 6. (a) Single fermion spectral functions near $\omega=0$ in pure AdS Reissner-Nordström (blue) and in the finite fermion density background (red). In the former the position of the maximum approaches $\omega=0$ as $T$ is lowered whereas in the latter the position of the maximum stays close to $T=0$ for all values of $T$. (b) Position of the maximum of the quasiparticle peak in $k-\omega$ plane, for different temperatures and $\Delta=5 / 4$. The probe limit around a AdS-RN black hole (blue) carries a strong temperature dependence of the $\omega_{\max }$ value, with $\omega_{\max , T \neq 0} \neq 0$. In the finite fermion density background, the position of the maximum (red) is nearly independent of temperature and stays at $\omega=0$.

"Dirac hair" is the preferred ground state. Through an analysis of the free energy, we argue that this gravitational solution with a non-zero fermion profile precisely corresponds to a system with a finite density of fermions. A spectral analysis still reveals a zoo of Fermi-surfaces in this ground state, but there are indications that in the full gravitationally backreacted solution only a Landau Fermi-liquid type Fermi surface survives. This follows in part from the relation between the spectral density and the Fermi momentum of a particular Landau liquid-like Fermi surface; it also agrees with the prediction from Luttinger's theorem. Furthermore, the spectral analysis in the finite density state shows no anomalous temperature dependence present in the pure charged black-hole single spectral functions. This also indicates that the finite density state is the true ground state.

The discovery of this state reveals a new essential component in the study of strongly coupled fermionic systems through gravitational duals, where one should take into account 
the expectation values of fermion bilinears. Technically the construction of the full gravitationally backreacted solution is a first point that is needed to complete our finding. A complete approach to this problem will have to take into account the many-body physics in the bulk. Within the approach presented in this paper, it means the inclusion of additional fermion wavefunctions, filling the bulk Fermi surface. The realization, however, that expectation values of fermion bilinears can be captured in holographic duals and naturally encode phase separations in strongly coupled fermion systems should find a large set of applications in the near future.

\section{Acknowledgments}

We thank F. Benini, J. de Boer, E. Gubankova, S. Hartnoll, C. Herzog, E. Kiritsis, H. Liu, M. Medvedyeva, J. McGreevy, A. O'Bannon, G. Policastro, and J. Sonner for extensive discussions. We are very grateful to the KITP Santa Barbara for its generous hospitality and the organizers and participants of the AdS/CMT Workshop (July 2009). KS thanks the Galileo Galilei Institute for Theoretical Physics for the hospitality and the INFN for partial support during the completion of this work. This research was supported in part by a VIDI Innovative Research Incentive Grant (K. Schalm) from the Netherlands Organization for Scientific Research (NWO), a Spinoza Award (J. Zaanen) from the Netherlands Organisation for Scientific Research (NWO) and the Dutch Foundation for Fundamental Research on Matter (FOM).

Open Access. This article is distributed under the terms of the Creative Commons Attribution Noncommercial License which permits any noncommercial use, distribution, and reproduction in any medium, provided the original author(s) and source are credited.

\section{References}

[1] C.M. Varma, Z. Nussinov and W. van Saarloos, Singular Fermi Liquids, Phys. Rept. 361 (2002) 267 [cond-mat/0103393].

[2] J.H. She and J. Zaanen, BCS Superconductivity in Quantum Critical Metals, Phys. Rev. B80 (2009) 184518 [arXiv:0905.1225].

[3] H. v. Löhneysen, A. Rosch, M. Vojta and P. Wölfle, Fermi-liquid instabilities at magnetic quantum phase transitions, Rev. Mod. Phys. 79 (2007) 1015 [cond-mat/0606317].

[4] M. Gurvitch and A.T. Fiory, Resistivity of La1.825Sr0.175CuO4 and Y Ba2Cu3O7 to 1100 K: Absence of saturation and its implications, Phys. Rev. Lett. 59 (1987) 1337.

[5] P. Phillips and C. Chamon, Breakdown of One-Paramater Scaling in Quantum Critical Scenarios for the High-Temperature Copper-oxide Superconductors, Phys. Rev. Lett. 95 (2005) 107002 [cond-mat/0412179].

[6] J. Zaanen, Quantum critical electron systems: the unchartered sign worlds, Science $\mathbf{3 1 9}$ (2008) 1205.

[7] J.M. Luttinger, Fermi Surface and Some Simple Equilibrium Properties of a System of Interacting Fermions, Phys. Rev. 119 (1960) 1153 [SPIRES]. 
[8] J.C. Campuzano, M.R. Norman and M. Randeria, Photoemission in the High- $T_{c}$ Superconductors, in Handbook of Physics: Physics of Conventional and Unconventional Superconductors, K.H. Benneman and J.B. Ketterson eds., Springer Verlag, (2004) [cond-mat/0209476].

[9] A. Damascelli, Z. Hussain and Z.-X. Shen, Angle-resolved photoemission studies of the cuprate superconductors, Rev. Mod. Phys. 75 (2003) 473 [cond-mat/0208504] [SPIRES].

[10] X.J. Zhou, T. Cuk, T. Devereaux, N. Nagaosa and Z.-X. Shen, Angle-Resolved Photoemission Spectroscopy on Electronic Structure and Electron-Phonon Coupling in Cuprate Superconductors in Handbook of High-Temperature Superconductivity: Theory and Experiment, J.R. Schrieffer, Springer Verlag, (2007) [cond-mat/0604284].

[11] H. Liu, J. McGreevy and D. Vegh, Non-Fermi liquids from holography, Phys. Rev. D 83 (2011) 065029 [arXiv:0903.2477] [SPIRES].

[12] M. Cubrovic, J. Zaanen and K. Schalm, String Theory, Quantum Phase Transitions and the Emergent Fermi-Liquid, Science 325 (2009) 439 [arXiv: 0904.1993] [SPIRES].

[13] T. Faulkner, H. Liu, J. McGreevy and D. Vegh, Emergent quantum criticality, Fermi surfaces and AdS2, Phys. Rev. D 83 (2011) 125002 [arXiv:0907.2694] [SPIRES].

[14] T. Faulkner, N. Iqbal, H. Liu, J. McGreevy and D. Vegh, From black holes to strange metals, arXiv:1003.1728 [SPIRES].

[15] T. Faulkner, N. Iqbal, H. Liu, J. McGreevy and D. Vegh, Strange metal transport realized by gauge/gravity duality, Science 329 (2010) 1043 [SPIRES].

[16] S.-S. Lee, A Non-Fermi Liquid from a Charged Black Hole: A Critical Fermi Ball, Phys. Rev. D 79 (2009) 086006 [arXiv: 0809.3402] [SPIRES].

[17] S.-J. Rey, String theory on thin semiconductors: Holographic realization of Fermi points and surfaces, Prog. Theor. Phys. Suppl. 177 (2009) 128 [arXiv:0911.5295] [SPIRES].

[18] S.S. Gubser, Breaking an Abelian gauge symmetry near a black hole horizon, Phys. Rev. D 78 (2008) 065034 [arXiv:0801.2977] [SPIRES].

[19] S.A. Hartnoll, C.P. Herzog and G.T. Horowitz, Building a Holographic Superconductor, Phys. Rev. Lett. 101 (2008) 031601 [arXiv: 0803.3295] [SPIRES].

[20] S.A. Hartnoll, C.P. Herzog and G.T. Horowitz, Holographic Superconductors, JHEP 12 (2008) 015 [arXiv: 0810.1563] [SPIRES].

[21] S.A. Hartnoll, Lectures on holographic methods for condensed matter physics, Class. Quant. Grav. 26 (2009) 224002 [arXiv:0903.3246] [SPIRES].

[22] F. Finster, J. Smoller and S.-T. Yau, Non-Existence of Black Hole Solutions to Static, Spherically Symmetric Einstein-Dirac Systems - a Critical Discussion, gr-qc/0211043 [SPIRES].

[23] F. Finster, J. Smoller and S.-T. Yau, Absence of stationary, spherically symmetric black hole solutions for Einstein-Dirac-Yang-Mills equations with angular momentum, Adv. Theor. Math. Phys. 4 (2002) 1231 [gr-qc/0005028] [SPIRES].

[24] F. Finster, J. Smoller and S.-T. Yau, The interaction of Dirac particles with non-Abelian gauge fields and gravity: Bound states, Nucl. Phys. B 584 (2000) 387 [gr-qc/0001067] [SPIRES]. 
[25] F. Finster, J. Smoller and S.-T. Yau, Non-existence of black hole solutions for a spherically symmetric, static Einstein-Dirac-Maxwell system, Commun. Math. Phys. 205 (1999) 249 [SPIRES].

[26] S.A. Hartnoll, J. Polchinski, E. Silverstein and D. Tong, Towards strange metallic holography, JHEP 04 (2010) 120 [arXiv:0912.1061] [SPIRES].

[27] S.A. Hartnoll and A. Tavanfar, Electron stars for holographic metallic criticality, Phys. Rev. D 83 (2011) 046003 [arXiv: 1008.2828] [SPIRES].

[28] J. de Boer, K. Papadodimas and E. Verlinde, Holographic Neutron Stars, JHEP 10 (2010) 020 [arXiv: 0907.2695] [SPIRES].

[29] X. Arsiwalla, J. de Boer, K. Papadodimas and E. Verlinde, Degenerate Stars and Gravitational Collapse in AdS/CFT, JHEP 01 (2011) 144 [arXiv:1010.5784] [SPIRES].

[30] L.-Y. Hung, D.P. Jatkar and A. Sinha, Non-relativistic metrics from back-reacting fermions, Class. Quant. Grav. 28 (2011) 015013 [arXiv: 1006.3762] [SPIRES].

[31] M. Cubrovic, Y. Liu, K. Schalm, Y.-W. Sun and J. Zaanen, Spectral probes of the holographic Fermi groundstate: dialing between the electron star and AdS Dirac hair, arXiv:1106.1798 [SPIRES].

[32] S.A. Hartnoll, D.M. Hofman and D. Vegh, Stellar spectroscopy: Fermions and holographic Lifshitz criticality, JHEP 08 (2011) 096 [arXiv: 1105.3197] [SPIRES].

[33] N. Iqbal, H. Liu and M. Mezei, Semi-local quantum liquids, arXiv:1105.4621 [SPIRES].

[34] N. Iqbal and H. Liu, Real-time response in AdS/CFT with application to spinors, Fortsch. Phys. 57 (2009) 367 [arXiv:0903.2596] [SPIRES].

[35] R. Contino and A. Pomarol, Holography for fermions, JHEP 11 (2004) 058 [hep-th/0406257] [SPIRES].

[36] E.M. Lifshitz and L.P. Pitaevskii, Statistical physics. Part 2, Pergamon Press, Oxford U.K. (1980).

[37] M. Čubrović, J. Zaanen and K. Schalm, Stability of the Fermi Liquid from holography, to appear.

[38] S. Sachdev, A model of a Fermi liquid using gauge-gravity duality, arXiv:1107.5321 [SPIRES].

[39] K. Goldstein, S. Kachru, S. Prakash and S.P. Trivedi, Holography of Charged Dilaton Black Holes, JHEP 08 (2010) 078 [arXiv:0911.3586] [SPIRES].

[40] T. Albash and C.V. Johnson, A Holographic Superconductor in an External Magnetic Field, JHEP 09 (2008) 121 [arXiv: 0804.3466] [SPIRES].

[41] R.-G. Cai, Z.-Y. Nie, B. Wang and H.-Q. Zhang, Quasinormal Modes of Charged Fermions and Phase Transition of Black Holes, arXiv: 1005.1233 [SPIRES]. 